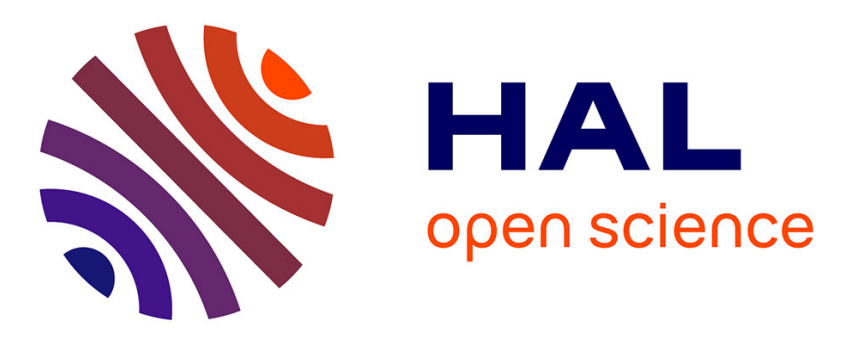

\title{
Three-dimensional numerical study of heat transfer and mixing enhancement in a circular pipe using self-sustained oscillating flexible vorticity generators
}

Samer Ali, Charbel Habchi, Sébastien Menanteau, T. Lemenand, Jean-Luc Harion

\section{To cite this version:}

Samer Ali, Charbel Habchi, Sébastien Menanteau, T. Lemenand, Jean-Luc Harion. Three-dimensional numerical study of heat transfer and mixing enhancement in a circular pipe using self-sustained oscillating flexible vorticity generators. Chemical Engineering Science, 2017, 162, pp.152-174. 10.1016/j.ces.2016.12.039 . hal-02525538

\section{HAL Id: hal-02525538 \\ https://univ-angers.hal.science/hal-02525538}

Submitted on 31 Mar 2020

HAL is a multi-disciplinary open access archive for the deposit and dissemination of scientific research documents, whether they are published or not. The documents may come from teaching and research institutions in France or abroad, or from public or private research centers.
L'archive ouverte pluridisciplinaire HAL, est destinée au dépôt et à la diffusion de documents scientifiques de niveau recherche, publiés ou non, émanant des établissements d'enseignement et de recherche français ou étrangers, des laboratoires publics ou privés. 


\title{
Three-dimensional numerical study of heat transfer and mixing enhancement in a circular pipe using self-sustained oscillating flexible vorticity generators
}

\author{
Samer Ali ${ }^{\mathrm{a}, \mathrm{b}, \mathrm{c}, *}$, Charbel Habchi $^{\mathrm{d}}$, Sébastien Menanteau ${ }^{\mathrm{e}}$, Thierry Lemenand ${ }^{\mathrm{f}}$, Jean-Luc Harion ${ }^{\mathrm{b}, \mathrm{c}}$ \\ ${ }^{a}$ Lebanese International University LIU, Mechanical Engineering Dept., Beirut, Lebanon \\ ${ }^{\mathrm{b}}$ Mines Douai, Industrial Energy Dept., Douai, France \\ ${ }^{\mathrm{C}}$ University of Lille Nord de France, Lille, France \\ ${ }^{\mathrm{d}}$ Notre Dame University - Louaize, Mechanical Engineering Dept., Zouk Mosbeh, Lebanon \\ e ICAM Lille, 59016, France \\ ${ }^{\mathrm{f}}$ LARIS, EA 7315, University of Angers, ISTIA, Angers, France
}

\section{A R T I C L E I N F O}

\section{Article history:}

Received 24 August 2016

Received in revised form 9 December 2016

Accepted 16 December 2016

Available online 31 December 2016

\section{Keywords:}

Fluid-structure interaction

Numerical simulation

Elastic flaps

Self sustained oscillations

Heat transfer

Mixing

Laminar flow

Three dimensional flow

HEV

\begin{abstract}
A B S T R A C T
In this paper, heat transfer and mixing performances are studied using three-dimensional numerical simulations of fluid-structure interactions. To this aim, a multifunctional heat exchanger/reactor geometry is investigated, consisting of a circular pipe where five arrays of four equally spaced trapezoidal vortex generators are inserted and inclined in a reversed position opposite to the flow direction with an angle of $45^{\circ}$ with respect to the pipe wall. A periodic rotation of $45^{\circ}$ is applied to the tabs arrays. Two cases are numerically studied: one using flexible vortex generators (FVG) that deform due to fluid forces applied on the structures and the other using conventional non deformable rigid vortex generators (RVG). For the FVG configuration, the tabs oscillate without addition of any external source of energy except that of the fluid flow itself, leading to a passive but dynamic way to perform vortex formation to disturb the flow. Both flow regimes are laminar with a constant Reynolds number of 1500 . The flow structures are analyzed using the proper orthogonal decomposition (POD) technique and the effect of tabs oscillation on vortices creation, suppression and dislocation is highlighted.

The effect of self-sustained free elastic tabs oscillation on heat transfer and mixing performances is numerically investigated by comparing the FVG with its corresponding RVG configuration. The Nusselt number comparison shows that the free tabs oscillation can improve the overall heat transfer of about $118 \%$ with respect to an empty pipe whereas it is about $97 \%$ for the RVG study. Finally, to assess the mixing performance, the transport of a passive scalar initially divided into two different concentrations in the pipe is numerically analyzed through the mixing index value. The FVG configuration shows a drastic improvement of the mixture quality at the exit of the pipe with an increase of $195 \%$ with respect to the RVG case, leading to much shorter and compact mixers and reactors.
\end{abstract}

(c) 2016 Elsevier Ltd. All rights reserved.

\section{Introduction}

Instabilities such as vortex-induced vibrations (VIV), galloping and fluttering occur when a fluid surrounding a structure supplies energy to the structure instead of absorbing it (Williamson and Govardhan, 2004; Huang, 1995). Usually, most of the studies in engineering disciplines intend to suppress such instabilities either by adding weights or by operating below a critical speed. When

\footnotetext{
* Corresponding author at: Lebanese International University LIU, Mechanical Engineering Dept., Beirut, Lebanon.

E-mail address: samer.ali@liu.edu.lb (S. Ali).
}

operating at this particular velocity, the instability indeed leads to self-sustained flapping motions of the structure in the flow and potentially causes damage to it. Recently some authors think about using this phenomenon and benefit from this flapping motion instead by harvesting electricity using patches of piezoelectric materials attached to the structure surface (Michelin and Doaré, 2013; Akcabay and Young, 2012).

In the particular context of multifunctional heat exchangers/ reactors, specific non deformable rigid vortex generators (RVG) are usually inserted in order to create secondary flows and disrupt the growth of the thermal boundary layer (Ahmed et al., 2012; Lei et al., 2010; Anxionnaz et al., 2008; Allison and Dally, 2007). These 


\begin{tabular}{|c|c|c|c|}
\hline \multicolumn{4}{|c|}{ Nomenclature } \\
\hline$a_{i}^{k}$ & POD mode coefficients $(-)$ & $R V G$ & rigid vortex generators \\
\hline$c$ & scalar $(-)$ & $S$ & maximum number of POD modes $(-)$ \\
\hline$c_{p}$ & specific heat $\left(\mathrm{J} \mathrm{kg}^{-1} \mathrm{~K}^{-1}\right)$ & $T$ & temperature $(\mathrm{K})$ \\
\hline CFD & computational fluid dynamics & $t$ & time $(s)$ \\
\hline CSD & computational structure dynamics & $\mathbf{u}$ & velocity vector $(u, v)\left(\mathrm{ms}^{-1}\right)$ \\
\hline $\mathbf{d}_{\mathbf{s}}$ & solid displacement vector $(\mathrm{m})$ & $\bar{U}_{f}$ & mean flow velocity $\left(\mathrm{ms}^{-1}\right)$ \\
\hline$d$ & pipe diameter $(\mathrm{m})$ & $v_{k}^{i}$ & $k^{\text {th }}$ component of the eigenvector (-) \\
\hline$D_{m}$ & mass diffusivity $\left(\mathrm{kg} \mathrm{m}^{-1} \mathrm{~s}^{-1}\right)$ & $(x, y)$ & Cartesian coordinate system (m) \\
\hline $\begin{array}{l}e \\
E\end{array}$ & $\begin{array}{l}\text { thickness of the elastic flap }(\mathrm{m}) \\
\text { Young's modulus }(\mathrm{Pa})\end{array}$ & $\begin{array}{l}(x, y) \\
()^{*}\end{array}$ & dimensionless position: $X^{*}=x / H, Y^{*}=y / H(-)$ \\
\hline$f$ & friction factor $(-)$ & () & row index: $A, B, C, D, E(-)$ \\
\hline$f_{e}$ & structural oscillation frequency $(\mathrm{Hz})$ & () & standard cardinal directions: $n, n e, e, s e, s, s w, w, n w(-)$ \\
\hline$f_{N}$ & structural natural frequency in vacuum $(\mathrm{Hz})$ & & \\
\hline$f_{v}$ & vortex shedding frequency $(\mathrm{Hz})$ & \multicolumn{2}{|c|}{ Greek symbols } \\
\hline $\mathbf{F}$ & deformation gradient tensor $(-)$ & $\gamma$ & mesh diffusion coefficient $(-)$ \\
\hline FVG & flexible vortex generators & $\epsilon$ & relative flow energy $(-)$ \\
\hline G & Green lagrangian strain tensor (-) & $\eta$ & thermal performance factor $(-)$ \\
\hline $\mathrm{GCI}$ & grid convergence index $(-)$ & $\lambda$ & eigenvalue $(-)$ \\
\hline$h_{g}$ & grid size $(\mathrm{m})$ & $v_{f}$ & fluid kinematic viscosity $\left(\mathrm{m}^{2} \mathrm{~s}^{-1}\right)$ \\
\hline$H$ & helicity field ( $\mathrm{m} \mathrm{s}^{-2}$ ) & $v_{s}$ & Poisson's ratio $(-)$ \\
\hline I & unity tensor $(-)$ & $\phi$ & normalized basis function $(-)$ \\
\hline$k_{t h}$ & thermal conductivity $\left(\mathrm{W} \mathrm{m}^{-1} \mathrm{~K}^{-1}\right.$ ) & $\rho$ & mass density $\left(\mathrm{kg} \mathrm{m}^{-3}\right)$ \\
\hline$L$ & length of the computational domain (m) & $\Sigma$ & Piola-Krichhoff stress tensor (-) \\
\hline$l$ & cell distance to the nearest moving boundary $(\mathrm{m})$ & $\zeta$ & cumulative relative flow energy (-) \\
\hline$\dot{m}$ & mass flow rate $\left(\mathrm{kg} \mathrm{s}^{-1}\right)$ & & \\
\hline MA & mean displacement amplitude (m) & \multicolumn{2}{|c|}{ Subscripts } \\
\hline MI & mixing index $(-)$ & ave & spatial average \\
\hline$N$ & total number of snapshots $(-)$ & $b$ & bulk \\
\hline $\mathrm{Nu}$ & Nusselt number $(-)$ & $f$ & fluid \\
\hline$p$ & pressure $(\mathrm{Pa})$ & $i$ & POD mode index \\
\hline$p_{c}$ & apparent order of convergence $(-)$ & $m$ & mesh \\
\hline $\operatorname{Pr}$ & Prandtl number $(-)$ & & solid \\
\hline$q^{\prime \prime}$ & 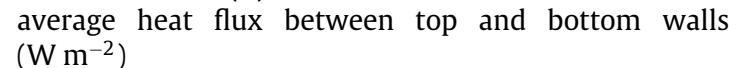 & $w$ & wall \\
\hline$r$ & grid refinement factor $(-)$ & & \\
\hline Re & Reynolds number (-) & & \\
\hline
\end{tabular}

RVGs are usually fixed and stay passive without any external control. Moreover, to add more flexibility to this technique, one can also think about making the vortex generators (VG) move by actively controlling the frequency and amplitude of oscillation of these VGs by relying on an external source of energy. This kind of study has been achieved for example by Lambert and Rangel (2010) who studied the effect of actively oscillating thin elastic flaps on fluid mixing in a microchannel. They found that the mixing is the greatest when one flap oscillates with the largest amplitude displacements. The addition of another flap also lead to the highest mixing performance when the flaps oscillate out of phase with an angle of $\pi / 2$. Furthermore, Yang and Chen (2008) numerically studied the effect of a transversely oscillating cylinder on heat transfer of heated blocks in a two-dimensional channel flow. They concluded that heat transfer is remarkably enhanced when the lock-in regime is reached, i.e. when the vortex shedding frequency synchronizes with the structural oscillation frequency. However, in these above-mentioned studies, the flaps oscillate using an external source of energy. Previous studies have furthermore put in evidence that self-sustained oscillations can be obtained by relying on the flow energy itself. Indeed, Ali et al. (2015) proposed an innovative concept using passive-dynamic control of flexible vortex generators (FVG) by creating an instability able to sustain the oscillatory motion in a two-dimensional channel. Their results show that the free elastic oscillations could improve the mixture quality up to $98 \%$ when comparing the corresponding configuration but using RVG instead. Moreover, the heat transfer displayed for the FVG case an increase up to $56 \%$ in the thermal performance and up to $134 \%$ in the overall heat transfer when compared to its relative RVG case. Furthermore, this fluidstructure interaction (FSI) problem caught the attention of several other authors. In fact, Shi et al. (2014) performed numerical simulations on a benchmark for FSI problems already available in the literature and proposed by Turek and Hron (2006), which consists of a two dimensional laminar flow around a flexible structure attached to a rigid cylinder. They included the effect of heat transfer to assess the thermal performance of the FSI benchmark and simulations were carried out at different Reynolds numbers calculated based on the rigid cylinder diameter $\left(R e_{D}=200-330\right)$. They observed that the FSI problem strengthens the disruption of the thermal boundary layer by vortex interaction with the wall and improves the mixing process between the hot and cold fluid regions. Their results indicate that VIV could increase the Nusselt number with a maximum enhancement of about $90.1 \%$ with respect to an empty channel. Nevertheless, the heat transfer effect on the same Turek and Hron benchmark has also been investigated by Soti et al. (2015) by varying the Prandtl number, the Reynolds number and the Young's modulus of the flap. The analyses suggest that larger Prandtl and Reynolds number tend to promote the thermal enhancement but with less efficiency. On the other hand, larger values of Young's modulus reduce the thermal enhancement and require much more pumping power. 
In light of the increased attention on the potential of applying FSI problems and self-sustained oscillations to heat transfer and mixing in real applications, we propose in this paper a threedimensional FSI study applied to a multifunctional heat exchanger/reactor configuration, namely the High-Efficiency-Vortex (HEV) geometry (Habchi et al., 2010a,b, 2012, 2013). It consists of five rows of trapezoidal vortex generators inserted as a $45^{\circ}$ rotated arrays in a pipe and inclined opposite to the flow direction with an angle of $45^{\circ}$ with respect to the pipe wall. The Reynolds number is held constant in the laminar regime with a value of 1500 based on the pipe diameter. The effect of using flexible vortex generators on heat transfer and mixing performances is numerically investigated by strongly coupling both fluid and structural solvers allowing FSI modeling. FVG and RVG cases are then compared by quantifying the Nusselt number, the thermal performance factor $\eta$ and finally the mixing index to evaluate the mixture quality. Heavy and computationally expensive simulation is performed on the FSI problem in order to complete $30 \mathrm{~s}$ of physical time. The flow structures of the FVG case are analyzed by applying the method of proper orthogonal decomposition proposed by Sirovich and Kirby (1987) and the behavior of vortex formation and patterns are compared to the RVG case. To this aim, the mathematical formulation and numerical procedure is first presented in Section 2. Section 3 presents the analyzed geometry and configurations through the description of the mesh and also initial and boundary conditions. The structural oscillations, flow patterns, heat transfer and mixing performances are next investigated through Sections 4 to 6 while Section 7 is eventually dedicated to concluding remarks.

\section{Mathematical formulation and numerical procedure}

In this study, a partitioned approach for solving the numerical fluid structure interaction problem is adopted. This method involves three solvers, one for fluid motion, another one for structural displacements and a third one for remeshing procedure. In addition, a strongly coupled solution is considered especially efficient when large structural displacements are present. This is achieved by introducing an additional FSI outer loop, where the solutions of the fluid and the structure are recalculated until reaching the convergence criteria set on forces and displacements (Le Tallec and Mouro, 2001). In this paper, this method is established by successively calling the ANSYS Fluent CFD-solver, and the ANSYS Mechanical CSD-solver (ANSYS Academic Research, 2015).

Thus, the flow field is governed by the unsteady Navier-Stokes equations for an incompressible viscous laminar flow. These equations are solved in a computational domain which deforms in time due to the flexible structure deformations. Therefore, an Arbitrary Lagrangian-Eulerian (ALE) formulation is used to solve the flow equations on a deforming mesh (Donea et al., 2004). The ALE formulation of the Navier-Stokes equations is written as:

$\nabla \cdot\left(\mathbf{u}_{\mathbf{f}}-\mathbf{u}_{\mathbf{m}, \mathbf{f}}\right)=0$

$\frac{\partial \mathbf{u}_{\mathbf{f}}}{\partial t}+\left(\mathbf{u}_{\mathbf{f}}-\mathbf{u}_{\mathbf{m}, \mathbf{f}}\right) \cdot \nabla \mathbf{u}_{\mathbf{f}}=-\frac{\nabla p}{\rho_{f}}+v_{f} \nabla^{2} \mathbf{u}_{\mathbf{f}}$

where $\left(\mathbf{u}_{\mathbf{f}}-\mathbf{u}_{\mathbf{m}, \mathbf{f}}\right)$ is the convective term with $\mathbf{u}_{\mathbf{f}}$ the fluid velocity and $\mathbf{u}_{\mathbf{m}, \mathbf{f}}$ the mesh motion velocity in the fluid domain. The pressure is denoted by $p$, the density of the fluid by $\rho_{f}$ and the kinematic viscosity by $v_{f}$. The Eulerian and Lagrangian descriptions are obtained by setting $\mathbf{u}_{\mathbf{m}, \mathbf{f}}=0$ or $\mathbf{u}_{\mathbf{m}, \mathbf{f}}=\mathbf{u}_{\mathbf{f}}$, respectively.

The mass transport equation of a scalar $c$ is considered to study the effect of the elastic flap deformations on fluid mixing:

$\frac{\partial c}{\partial t}+\left(\mathbf{u}_{\mathbf{f}}-\mathbf{u}_{\mathbf{m} . \mathbf{f}}\right) \cdot \nabla c=D_{m} \nabla^{2} c$ where $D_{m}$ is the mass diffusivity of the scalar $c$. Here, the convective scalar mixing is only considered so mass diffusion term is set to 0 .

The heat equation is also solved in the fluid domain and is given by:

$\frac{\partial T_{f}}{\partial t}+\left(\mathbf{u}_{\mathbf{f}}-\mathbf{u}_{\mathbf{m} . \mathbf{f}}\right) \cdot \nabla T_{f}=\frac{k_{t h}}{\rho_{f} C_{p}} \nabla^{2} T_{f}$

where $T_{f}$ is the fluid temperature, $k_{t h}$ is the thermal conductivity and $C_{p}$ is the specific heat.

The equation of motion for an elastic isothermal solid structure can be described from a Lagrangian point of view, i.e. in terms of the initial configuration at $t=0$, and it reads:

$\rho_{s} \frac{\partial^{2} \mathbf{d}_{s}}{\partial t^{2}}=\nabla \cdot\left(\Sigma \cdot \mathbf{F}^{\mathbf{T}}\right)+\rho_{s} f_{b}$

where $\mathbf{d}_{\mathbf{s}}$ is the displacement of the structure, $f_{b}$ is the resulting body force, $\rho_{s}$ is the density of the structure and $\mathbf{F}$ is the deformation gradient tensor given by:

$\mathbf{F}=\mathbf{I}+\nabla \mathbf{d}_{\mathbf{s}}^{\mathbf{T}}$

where $\mathbf{I}$ is the identity.

The second Piola-Kirchhoff stress tensor $\Sigma$ is related to the Green Lagrangian strain tensor G following (Tukovic and Jasak, 2007; Bos, 2010):

$\Sigma=2 \mu_{s} \mathbf{G}+\lambda_{s} \operatorname{tr}(\mathbf{G}) \mathbf{I}$

with $\mathbf{G}$ given by:

$\mathbf{G}=\frac{1}{2}\left(\mathbf{F}^{\mathbf{T}} \cdot \mathbf{F}-\mathbf{I}\right)$

Here $t r$ is the tensor trace, $\lambda_{s}$ and $\mu_{s}$ are Lamé constants which are characteristics of the elastic material. They are linked to the Young modulus $E$ and Poisson's coefficient $v_{s}$ by:

$\lambda_{s}=\frac{v_{s} E}{\left(1+v_{s}\right)\left(1-2 v_{s}\right)}$

$\mu_{s}=\frac{E}{2\left(1+v_{s}\right)}$

Fluid-structure interaction problems with moving boundaries require a third coupled solver for automatic internal mesh motion. The mesh motion solver in Fluent consequently deforms the internal fluid domain while maintaining the quality and validity of the deforming mesh. In this case, the displacement of the fluidstructure interface, which is the result of the structural solver, is then used as a boundary condition for the mesh motion solver. The present study employs the Laplace smoothing equation given by the following expression:

$\nabla \cdot\left(\gamma \nabla \mathbf{u}_{\mathbf{m}}\right)=0$

where $\gamma$ is the mesh diffusion coefficient and $\mathbf{u}_{\mathbf{m}}$ the mesh displacement velocity. The mesh diffusion coefficient used in this study is a function of the boundary distance:

$\gamma=\frac{1}{l^{\alpha}}$

where $l$ is a normalized boundary distance. A diffusion parameter $\alpha$ of 1.5 has been used in the present study, which preserves larger regions of the mesh near the moving boundary, and cause the regions away from the moving boundary to absorb more of the motion.

To avoid divergence problems especially caused by negative cell volumes, the mesh solver supports several remeshing methods. The one used in this study is the local cell remeshing that applies to tetrahedral cells in the domain. This method agglomerates cells 
that violate the skewness or size criteria and locally remeshes the agglomerated cells. If the new cells or faces satisfy the skewness criterion, the mesh is locally updated with the new cells (with the solution interpolated from the old cells) (ANSYS Academic Research, 2015). Otherwise, the new cells are discarded and the old cells are retained. In the present study a maximum cell skewness of 0.7 has been used for remeshing with cell size criterion depending on the minimum and maximum length scale of the mesh.

In order to perform the load and motion transfer between the different meshes for fluid and structure, the General Grid Interface (GGI) mapping algorithm is used (Galpin et al., 1995). At each time step, the FSI iteration loop sequentially calls the structural solver, the mesh motion algorithm and then the fluid solver until the convergence limits for displacements and forces are reached. An under-relaxation factor for both displacement and force is set to unity for all the simulations performed and convergence criteria set to $10^{-4}$ on force and displacement.

The pressure-velocity coupling is established using the Coupled algorithm (ANSYS Academic Research, 2015), which solves the momentum and pressure-based continuity equations together. The convergence criteria for pressure, velocity, energy and scalar is set to $10^{-6}$. The Laplace mesh smoothing convergence criterion is set to $10^{-4}$. Temporal discretization is performed using a first order implicit scheme and the time step used for all the present simulations is set to $5 \times 10^{-4} \mathrm{~s}(\approx 400$ time steps per period of oscillation).

Eventually, only the ANSYS Fluent CFD-solver is activated for the numerical simulations carried out with rigid vortex generators, with the above mentioned parameters kept identical.

The overall procedure described above has already been validated in previous studies in two-dimensional flow configurations and more details can be found in Ali et al. (2015). Moreover, taking into account the third component in the spanwise direction of the flow must not modify conclusion regarding the validation of the numerical solvers and coupling.

\section{Problem description}

The computational domain consists of a three-dimensional flow in a circular pipe where vortex generators are placed along the walls. In this section, we present the studied configurations and associated boundary and initial conditions, followed by a mesh validation study.

\subsection{Problem description: geometries, initial and boundary conditions}

In the present study, an HEV-like geometry is investigated as shown in Fig. 1. It consists of a round pipe with a diameter $d=54 \mathrm{~mm}$ in which five arrays (A, B, C, D, E) of FVG are inserted. Each array is composed of four diametrically opposed trapezoidal VG inclined to the wall at an angle $\beta=45^{\circ}$ and directed inversely to the main flow direction. Each trapezoidal VG has a height of $h=16 \mathrm{~mm}$, a base length of $b=15 \mathrm{~mm}$, an opposite parallel side to the base with a length $a=10 \mathrm{~mm}$ and a thickness $e=0.2 \mathrm{~mm}$. The distance between two successive FVG arrays is $30 \mathrm{~mm}$. It should be noted that arrays $B$ and $D$ undergo a $\theta=45^{\circ}$ tangential rotation with respect to array A. The FVG orientations of the arrays $\mathrm{A}, \mathrm{C}$ and $\mathrm{E}$ follow a cardinal direction notation commonly denoted by their initials $n$ for north, $e$ for east, $s$ for south and $w$ for west. On the other hand, FVG orientations of the arrays B and D follow similarly the notation ne for northeast, se for southeast, $s w$ for southwest and $n w$ for northwest. Thus, every FVG can be located by its row letter followed by its cardinal direction. For example, FVG An points to the tab in the first row $A$ and at the top position (north $n$ ). The FVG configuration is further compared to its relative RVG case in terms of flow structures, heat transfer and mixing quality.

No slip boundary conditions are set at the pipe wall. The outlet is set at zero pressure and Neumann zero for velocity. A parabolic laminar velocity profile corresponding to a fully developed laminar flow is set at the flow inlet:

$U_{f, \text { inlet }}=2 \bar{U}_{f, \text { inlet }}\left[1-\left(\frac{4\left(x^{2}+y^{2}\right)}{d^{2}}\right)\right]$

where $\bar{U}_{f, \text { inlet }}$ is the mean flow velocity at the inlet.

To characterize the mixing process, the fluid domain is initially divided into two equal parts: at $x>0, c$ is set to 1 and at $x<0, c$ is set to 0 as shown in Fig. 1(b). Zero flux boundary conditions are prescribed for the mass transport equation along the walls and the rigid/flexible flaps.

To characterize the heat transfer process, the same conditions are set for both FVG and RVG cases with a uniform wall temperature $T_{w}=360 \mathrm{~K}$ imposed at the tube wall. The laminar parabolic velocity profile is prescribed at the inlet with a uniform inlet temperature $T_{i n}=300 \mathrm{~K}$. The rigid/flexible tabs are treated as insulating material with zero heat flux along them.

Finally, the physical properties of the FVGs/RVGs and the flow conditions are summarized in Table 1.

\subsection{Grid size independence study}

The three dimensional FSI problem is very computationally expensive to run and considering to do a mesh validation study is not reasonable (for instance, the FSI simulation ran during seven months to bypass the transitional state from initial conditions to periodic state and finally reached $30 \mathrm{~s}$ physical time modeled). For this reason the mesh study is thus conducted on simulations of the RVG case and the final retained mesh is used for both unsteady FVG and RVG cases. The fluid domain is discretized with tetrahedral cells using three different mesh densities, refined at the pipe wall and at the fluid-structure interface, i.e. the elastic/rigid tabs. The three mesh densities and their main characteristics are summarized in Table 2.

To determine the appropriate mesh density for grid size independence, the simulations are performed on increasing mesh densities. The mesh validity verification is based on the method proposed by Celik et al. (2008) where the grid convergence index (GCI) and the apparent order of convergence $\left(p_{c}\right)$ can be obtained. Since the heat transfer strongly depends on the near-wall refinement, the Nusselt number $\mathrm{Nu}$ is chosen for the mesh validity verification and is expressed as:

$N u_{z}=\frac{q_{w}^{\prime \prime}(z) d}{k\left(T_{w}-T_{b}(z)\right)}$

where $q_{w}^{\prime \prime}$ is the averaged heat flux along a circle that has a diameter equal to that of the pipe at a position $z, T_{w}$ the temperature along the walls, $k$ is the thermal conductivity of air and $T_{b}(z)$ is the local bulk temperature defined by:

$T_{b}(z)=\frac{\int_{A} U(x, y, z) T(x, y, z) d A}{\int_{A} U(x, y, z) d A}$

where $A$ represents the local cross-section of the pipe.

The results obtained from applying the method of Celik et al. (2008) are summarized in Table 3, where $\phi_{\text {refined }}$ is the variable calculated from the solution of the refined mesh, $\phi_{e x t}$ is the extrapolated variable and $e_{a}^{23}$ is the relative error between Mesh 3 and Mesh 2. It can be concluded from Table 3 that the uncertainty in the fine-grid solution is about $0.59 \%$ for the integrated Nusselt number (along all the pipe length $L=0.4 \mathrm{~m}$ ). The refined Mesh 3 

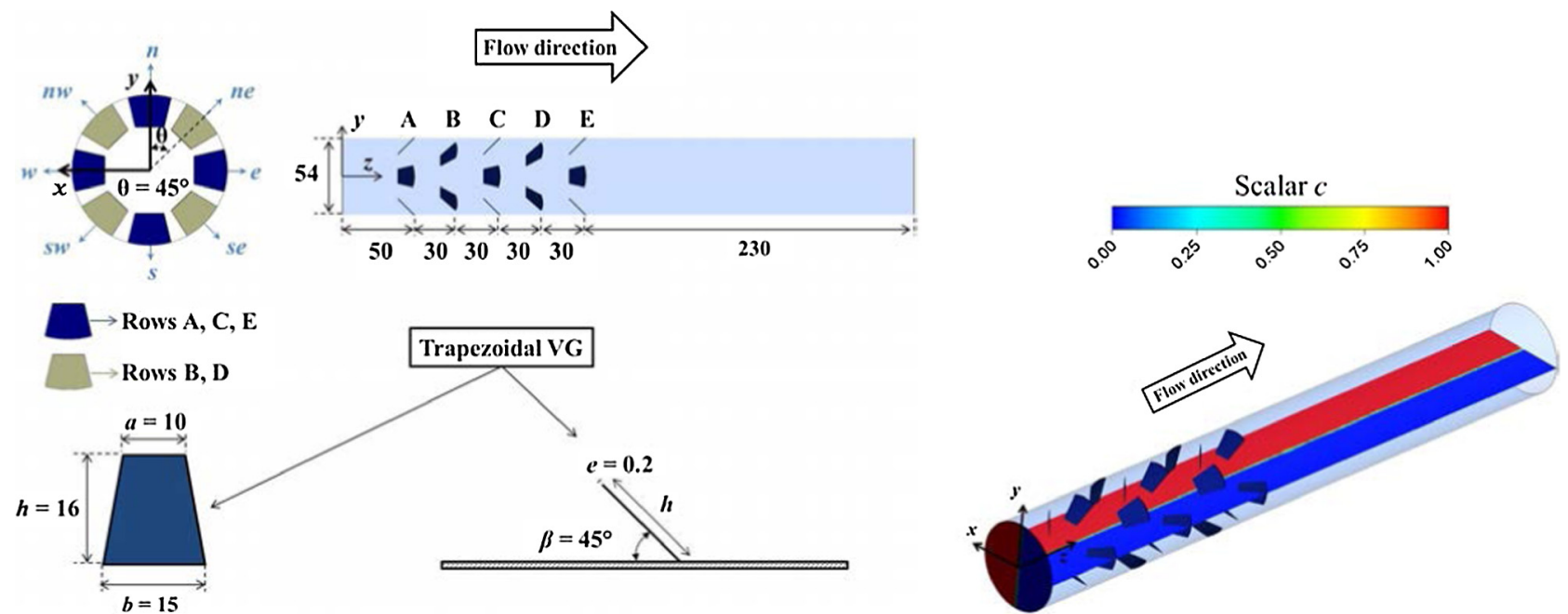

(a)

(b)

Fig. 1. HEV geometry: (a) tab dimensions (in $\mathrm{mm}$ ) and (b) initial scalar field distribution in the fluid domain.

Table 1

Physical parameters and flow conditions for all cases.

\begin{tabular}{ccccc}
\hline Cases & $\rho_{s}\left(\mathrm{~kg} / \mathrm{m}^{3}\right)$ & $v_{s}$ & $E(\mathrm{~Pa})$ & $R e_{d}=\left(\bar{U}_{f, \text { inlet }} d\right) / v_{f}$ \\
\hline FVG & 1000 & 0.45 & $1 \times 10^{6}$ & 1500 \\
RVG & 1000 & - & - & 1500 \\
\hline
\end{tabular}

Table 2

Global meshes characteristics used for grid size independance study.

\begin{tabular}{cccc}
\hline & Mesh 1 & Mesh 2 & Mesh 3 \\
\hline Initial number of cells (N) & 367,820 & 810,175 & $1,845,922$ \\
Grid size $h_{g}=\left[\frac{1}{N} \sum_{i=1}^{N}\left(\Delta V_{i}\right)\right]^{\frac{1}{3}}$ & $13.55 \times 10^{-4}$ & $10.42 \times 10^{-4}$ & $7.91 \times 10^{-4}$ \\
Grid refinement factor & - & $h_{2} / h_{1}=1.30$ & $h_{3} / h_{2}=1.32$ \\
$r=h_{i} / h_{j}$ & & & \\
\hline
\end{tabular}

where $\Delta V_{i}$ is the volume of the $i^{\text {th }}$ cell.

Table 3

Estimated order of convergence $p_{c}$ and GCI for refined Mesh 3.

\begin{tabular}{cccccc}
\hline Parameter & $\phi_{\text {refined }}$ & $\phi_{\text {ext }}$ & $e_{a}^{23}(\%)$ & $p_{c}$ & GCI (\%) \\
\hline$\frac{1}{L} \int_{0}^{L} N u_{z} d z$ & 17.55 & 17.6 & 0.85 & 3.75 & 0.59 \\
\hline
\end{tabular}

is thus considered as a reliable reference mesh density for the FVG and RVG simulations.

\section{Results and discussion}

In this section, the structural oscillation behavior in the FVG case is presented, then the flow analysis is based on the proper orthogonal decomposition technique to identify the different vortex modes. In addition, we compare the FVG case to its relative RVG case in order to identify the effect of structural oscillations on heat transfer and mixing performances.

\subsection{Structural oscillations}

The displacement of the tip position of every tab is first depicted in the $z$ direction. The tab oscillation amplitudes actually increase with time until reaching a quasi-periodic regime at time $t>19 \mathrm{~s}$ displaying a sinusoidal pattern of oscillation. For clarity reasons, only the last second of simulation is presented in Fig. 2. From this temporal signals, we can see that at rows $B$ and $D$, the tabs almost show no significant oscillations whereas at rows A, C and E, the tabs undergo large displacement amplitudes. Since the rows B and $\mathrm{D}$ undergo a $45^{\circ}$ tangential rotation, the deformations of tabs in rows $\mathrm{A}, \mathrm{C}$ and $\mathrm{E}$ have small influence on their motions. Thus the interaction of tabs between two rows $B$ and $D$ is apparently weaker than the interaction of tabs in three rows $A, C$ and $E$.

Fig. 3 illustrates then the deformation cycle of tabs during one cycle of oscillations. The wireframes are used to indicate the initial fixed position of the tabs before any fluid forces applied. In fact, as the tabs An and As reach their minimum amplitudes and tabs Ae and Aw reach their maximum amplitudes as shown in Fig. 3(b), the flow is now oriented in the region from $s$ to $n$ and blocked in the region from $e$ to $w$. Consequently, the drag forces will be concentrated on the wake region, especially at row $\mathrm{C}$ and at tabs $\mathrm{Cn}$ and $C s$, since the trajectory of flow to $\mathrm{Cw}$ and $\mathrm{Ce}$ is blocked by the upstream tabs Aw and Ae. Nevertheless, when the tabs Aw and Ae reach their highest position as shown in Fig. 3(b), their stored kinetic energy will make them revert backward with a motion opposed to that of the flow to reach their minimum amplitude as shown in Fig. 3(e). Hence, this will allow the flow to exert forces at tabs $\mathrm{C} w$ and $\mathrm{Ce}$. The same holds true when analyzing rows $\mathrm{C}$ and $\mathrm{E}$. Moreover, due to this drafting effect and this alternating behavior in subjecting every tab to high/low loads, a phase shift is produced between the rows. Indeed, tabs in row $\mathrm{C}$ oscillate out of phase with tabs in row A. Similarly, tabs of row E oscillate out of phase with tabs of row $C$ and as a result are in phase with tabs of row A. The amplitude of oscillations are summarized in Table 4. Since the displacements of rows B and D are relatively small (order of $10^{-4} \mathrm{~m}$ ), we only present results corresponding to tabs in rows A, C and E. Since the signal of the displacement is quasi-periodic, the mean amplitude MA of flap E can moreover be expressed by:

$\mathrm{MA}=\frac{1}{N_{\text {peak }}} \sum_{i=1}^{N_{\text {peak }}}\left|z_{i_{\text {peak }}}-\bar{z}\right|$

where $N_{\text {peak }}$ is the number of maximum and minimum peaks in the signal, $z_{i_{\text {peak }}}$ are the individuals maximum and minimum values and $\bar{z}$ is the mean displacement. The amplitude calculation is performed from $t_{1}=19 \mathrm{~s}$ till $t_{2}=30 \mathrm{~s}$ in order to omit the transition period in 


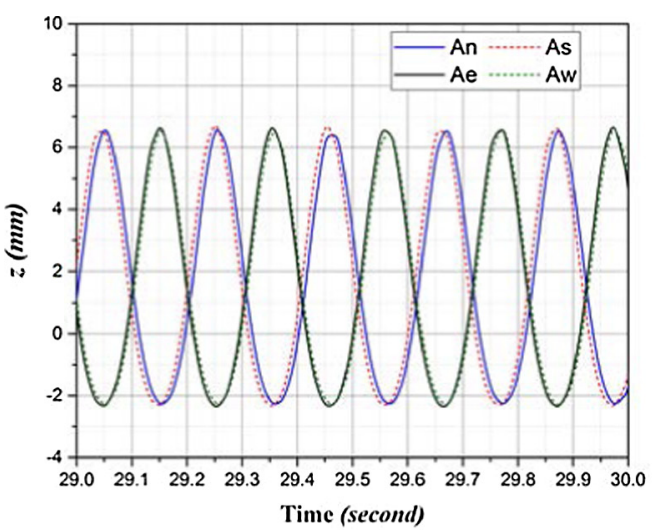

(a)

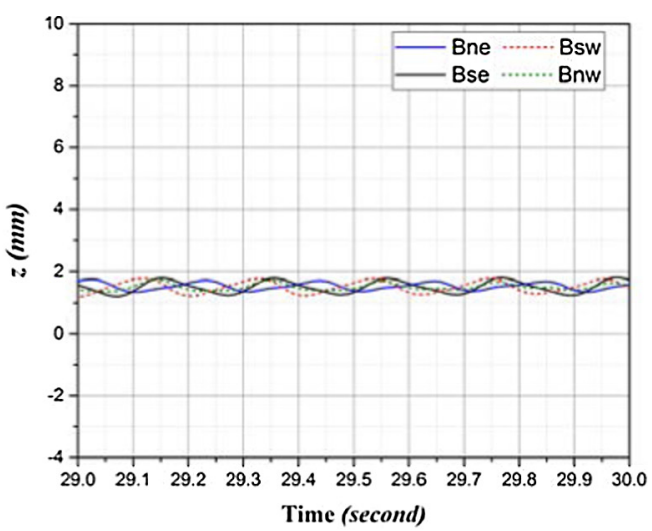

(b)

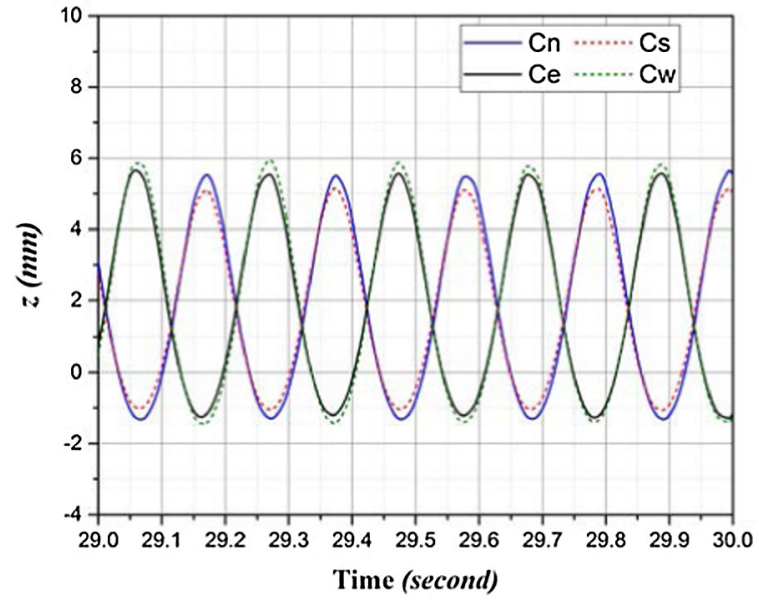

(c)

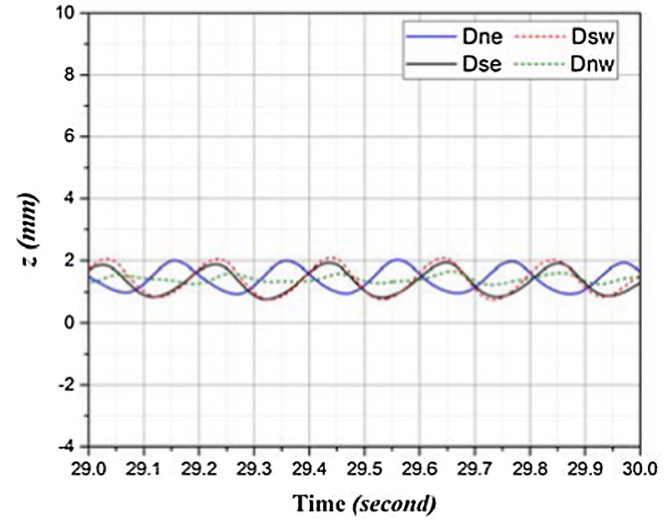

(d)

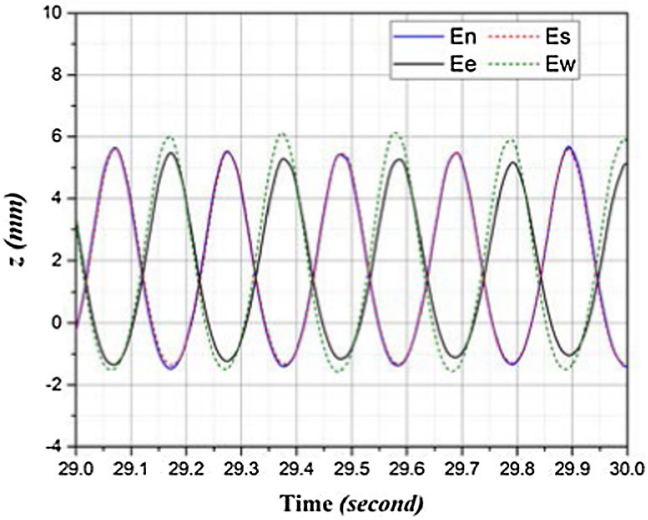

(e)

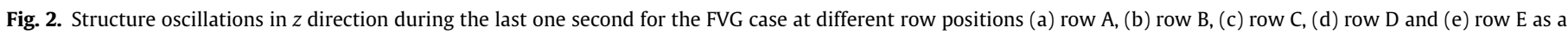
function of time.

the signal. It is important to note that tabs of the first row A experience the highest amplitude oscillations since the first row is subjected to the highest drag forces and the rows downstream experience a drafting effect and consequently a reduction in drag. However, the tabs of row E undergo larger amplitude oscillations than tabs of row $C$, this could be explained by the fact that the motions of tabs of row E are unrestricted nor influenced by further downstream tabs.

In order to calculate the phase shift angle of every tab calculated with respect to tab An, we compute the Fast Fourier Transformation (FFT) on the cross-correlation of the two signals of interest. Then the phase angle identified at the highest frequency peak can be considered as the phase shift. The results are summarized in Table 5: if the phase angle is negative, it means that tab An is lagging, otherwise $A n$ is leading. It can be shown that the tabs in row A oscillate out of phase with the tabs in rows $C$ and approximately in phase with the tabs in rows E. Moreover, in a given row, the tabs located at the cardinal direction $n$ and $s$ oscillate out of phase with the tabs at the cardinal directions $e$ and $w$. For example, in row A, the tabs An lags tabs $\mathrm{A} e$ and $\mathrm{A} w$ with a phase difference of $173.53^{\circ}$ and $167.4^{\circ}$ respectively. However, tab An approximately oscillate in phase with the south relative tab As with a small phase difference of $6.8^{\circ}$. 


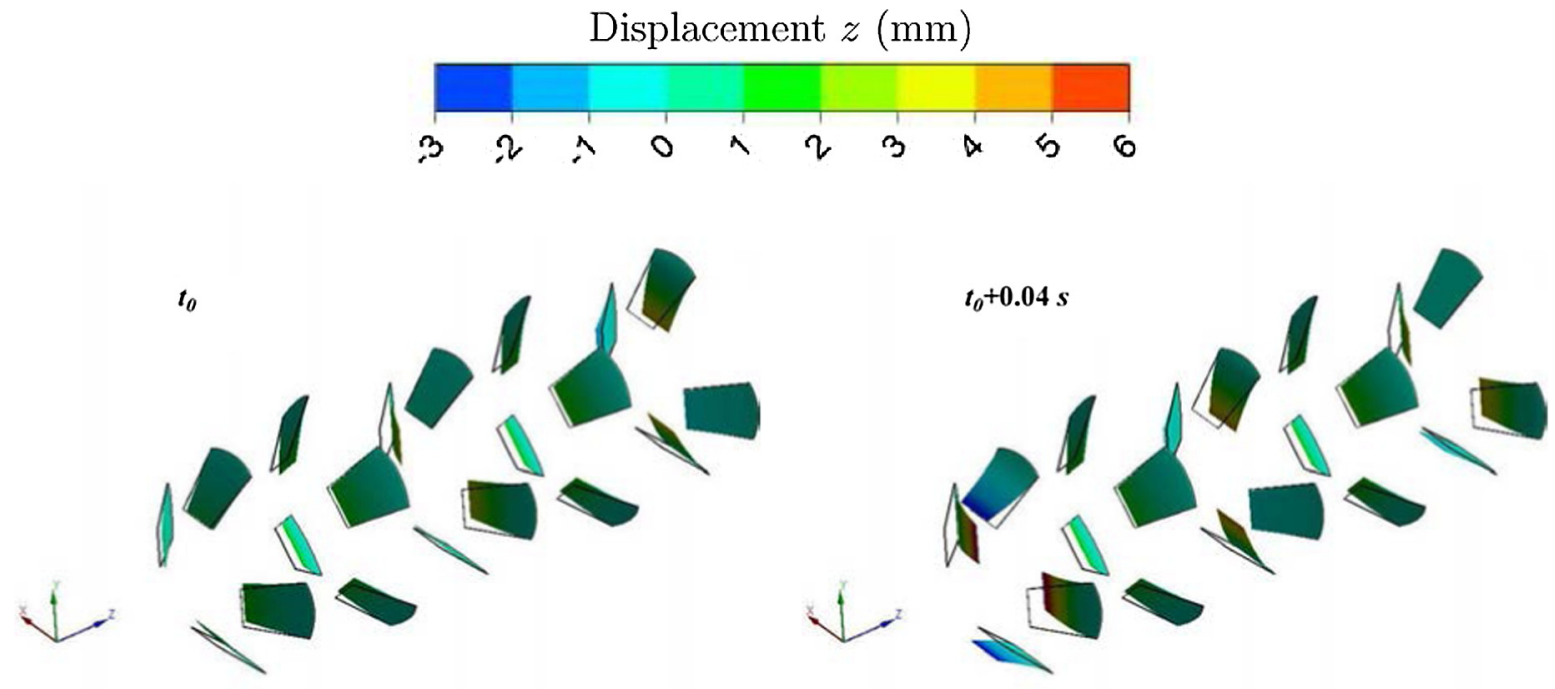

(a)

(b)
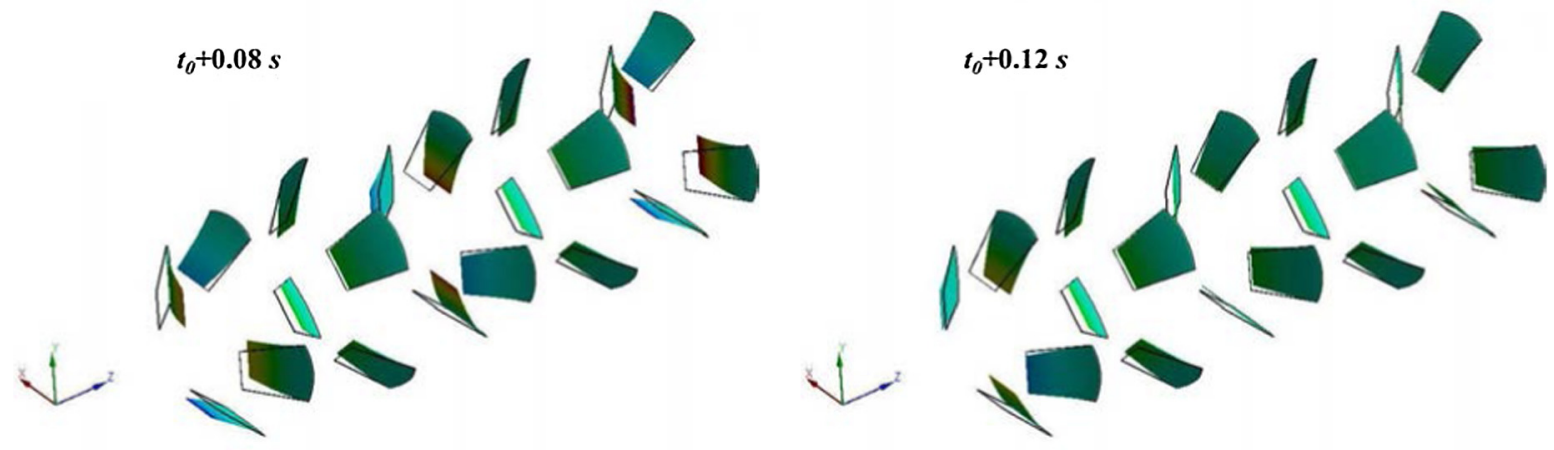

(c)

(d)

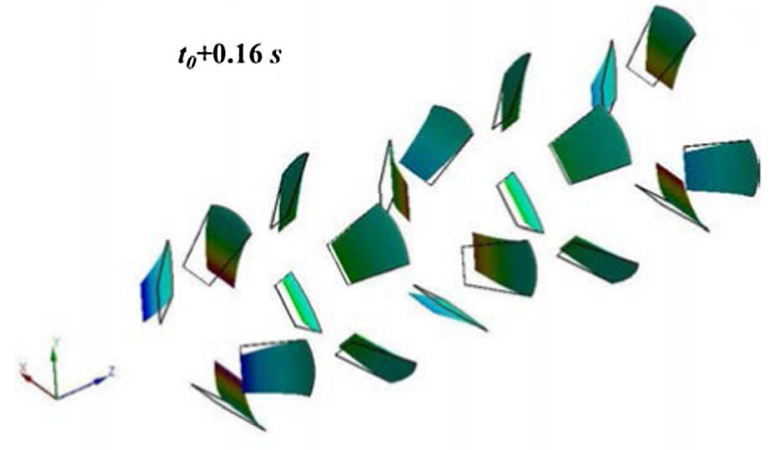

(e)

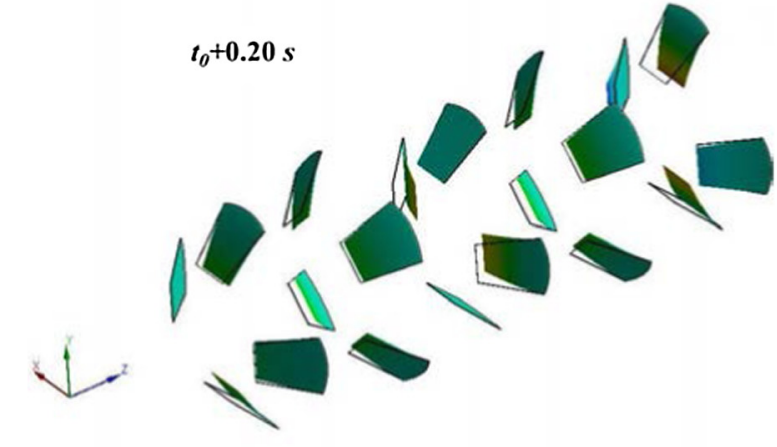

(f)

Fig. 3. 3D perspective view of tabs deformations during one period of oscillation; the fixed wireframe represents the initial position of every tab.

Finally we compute the natural frequency of the trapezoidal tabs by using the Block Lanczos mode extraction method (ANSYS Academic Research, 2015). The natural frequency $f_{N}$ is equal to $5.1(\mathrm{~Hz})$. An important feature is that the structural oscillation frequencies of all the tab appear to be really close to the natural frequency with a value $f_{e}=4.86(\mathrm{~Hz})$, leading to a resonance effect.
This resonance effect will synchronize with the frequency of appearance of the vortex modes $f_{v}$ and will be demonstrated in Section 4.2 using the proper orthogonal decomposition technique to analyze the flow. The FFT on the displacement signal is presented in Fig. 4 to clearly identify a peak oscillation frequency close to the fundamental frequency. The tabs in the three rows $\mathrm{A}, \mathrm{C}$ and $\mathrm{E}$ 
Table 4

Mean oscillation amplitudes MA (in $\mathrm{mm}$ ) in the $z$ direction for tabs rows A, C and E.

\begin{tabular}{ccccc}
\hline Row & $n$ & $e$ & $s$ & $w$ \\
\hline A & 4.33 & 4.45 & 4.43 & 4.33 \\
C & 3.43 & 3.42 & 3.17 & 3.51 \\
E & 3.79 & 3.58 & 3.59 & 3.63 \\
\hline
\end{tabular}

Table 5

Phase angle in degrees for tabs rows $\mathrm{A}, \mathrm{C}$ and $\mathrm{E}$.

\begin{tabular}{ccccc}
\hline Row & $n\left(^{\circ}\right)$ & $e\left(^{\circ}\right)$ & $s\left({ }^{\circ}\right)$ & $w\left(^{\circ}\right)$ \\
\hline A & 0 & -173.53 & 6.8 & -167.4 \\
C & -135.85 & 32.32 & -138.96 & 32.11 \\
E & 50.75 & -131.83 & 48.5 & -140.77
\end{tabular}

display higher power spectrum density (PSD) peaks than those located in rows B and D at the fundamental frequency. This is due to the fact that the energy transfer between the fluid and the tabs at locations A, C and E is more significant and leads eventually in larger displacement amplitudes than for rows B and D. However, with a lower number of rows that are tangentially rotated as rows $B$ and $D$ (as opposed to three non rotated rows $A, C$ and $E$ ), the interaction and energy transfer between the tabs and the fluid forces is smaller and results in lower PSD peaks as shown in Fig. 4(b) and (d), which eventually leads to smaller oscillation amplitudes.

\subsection{Flow patterns and proper orthogonal decomposition (POD)}

Fig. 5 shows the helicity distribution at different row positions for the RVG case, typically downstream of row A in Fig. 5(a), row C in Fig. 5(b) and row E in Fig. 5(c). The helicity $(H=\mathbf{u} \cdot \mathbf{w})$ is defined as the dot product of the velocity vector $\mathbf{u}$ and the vorticity vector $\mathbf{w}$.

Trapezoidal vortex generators when set at a certain angle relative to the pipe wall can create a pressure difference between the high momentum area of the core fluid and the low momentum area downstream in the wake region. This effect initiates a swirling motion that gives birth to a counter-rotating vortex pair (CVP) denoted at index positions 1 and 2 as in Fig. 5(a)-(c). Secondary counter-rotating vortices are also observed at index positions 3 , 4,5 and 6 , above and below every primary CVP. These vortices are caused by the interaction of the high radial velocity induced by the primary CVP with the stagnant fluid region near the wall (index positions 3 and 4) or with the low momentum region at the wake of the tab (index position 5 and 6). In addition to the longitudinal vortices, transverse vortices are also formed whose axis of rotation is perpendicular to the flow direction. Owing to the pressure difference across the tab, the flow wraps around and deflects inwards from the tab sides creating these transverse vortices.

Fig. 5(b) shows the helicity distribution downstream row $\mathrm{C}$. The secondary vortices 5 and 6 have reached this row $C$ after being convected from the previous row $B$. Hence, after each passage of arrays, the vortices increase in both size and strength. Moreover, we can observe the merging process of these secondary vortices with the primary CVP downstream the last row E (Fig. 5(c)) to form one large CVP that scales up to the trapezoidal tabs height. Fig. 5(d) illustrates then the streamlines and vectors of the velocity field in the wake of tab An. The flow is deflected upwards and over the tip of the tab, since the stagnation pressure in front of the tab is higher than the pressure above the free end. In particular, due to the lower pressure behind the tab, the flow over the top experiences downwash in the wake and results in a reversed vortex motion.
These recirculation vortices are considered bad for mixing and heat transfer since the fluid will stay trapped in the wake region and the only transport phenomenon that will be acting is diffusion.

In order to analyze the complex flow structures of the FVG case, we use the proper orthogonal decomposition technique. The present analysis uses the so called "Snapshot POD" which is introduced by Sirovich and Kirby (1987). Each time instance is considered to be a snapshot of the flow. The analysis is performed on 1500 snapshots on each of the three planes previously introduced in the RVG case (i.e. plane 1 at $z / d=1.11$, plane 2 at $z / d=2.22$ and plane 3 at $z / d=3.33$ ). The analysis is based on the helicity scalar field $\left(H_{j}^{k}\right)$, where index $k$ runs through the $N$ snapshots and $j$ runs through the $M$ positions of the helicity field in a given snapshot (i.e. $H_{j}=H\left(x_{j}, y_{j}, z_{j}\right)$ ). All the helicity components from the $N$ snapshots are further arranged in a matrix $\mathbf{H}$ as follows:

$\mathbf{H}=\left[\begin{array}{llll}H^{1} & H^{2} & \ldots & H^{N}\end{array}\right]=\left[\begin{array}{cccc}H_{1}^{1} & H_{1}^{2} & \cdots & H_{1}^{N} \\ H_{2}^{1} & H_{2}^{2} & \cdots & H_{2}^{N} \\ \vdots & \vdots & \vdots & \vdots \\ H_{M}^{1} & H_{M}^{2} & \cdots & H_{M}^{N}\end{array}\right]$

Thus, the autocovariance matrix can be created as:

\section{$\widetilde{\mathbf{C}}=\mathbf{H}^{T} \mathbf{H}$}

The goal of the POD technique is to find a sequence of orthonormal basis functions $\phi^{i}$ (POD modes) by solving the eigenvalue problem of the autocovariance matrix $\widetilde{\mathbf{C}}$ as:

$\widetilde{\mathbf{C}} \mathbf{v}^{i}=\lambda^{i} \mathbf{v}^{i}$

Then according to the size of the eigenvalues, the solutions are arranged in decreasing order as:

$\lambda^{1}>\lambda^{2}>\cdots>\lambda^{N} \geqslant 0$

The basis functions $\phi^{i}$ are obtained by projecting $\mathbf{H}$ onto the eigenvectors $\mathbf{v}$ of Eq. (19) with subsequent normalization, defined by:

$\phi^{i}=\frac{\sum_{k=1}^{N} v_{k}^{i} H^{k}}{\left\|\sum_{k=1}^{N} v_{k}^{i} H^{k}\right\|}, \quad i=1, \ldots, N$

where $v_{k}^{i}$ is the $k^{\text {th }}$ component of the eigenvector corresponding to $\lambda^{i}$ from Eq. (19). The basis functions represent extracted flow patterns which are considered as coherent structures in the literature.

Each snapshot can be reconstructed from a series of POD modes with expansion coefficients $a_{i}$ associated with each POD mode. The coefficients of each mode are computed by projecting the original helicity field onto the computed basis functions:

$\mathbf{a}^{k}=\psi^{T} H^{k}$

where $\psi=\left[\begin{array}{llll}\phi^{1} & \phi^{2} & \ldots & \phi^{N}\end{array}\right]$ is introduced. Thus, any snapshot can be represented as a linear combination of the basis functions as follows:

$H^{k}=\sum_{k=1}^{S} a_{i}^{k} \phi^{i}$

where $S$ is the number of modes necessary for modeling the characteristics of the large scale data as a low dimensional model.

The POD coefficients $\mathbf{a}^{k}$ indicate how much a POD mode contributes in each time snapshot. The eigenvalues $\lambda^{i}$ are proportional to the flow energy in the flow and by sorting them in a decreasing order as in Eq. (20), the most energetic modes can be identified. 


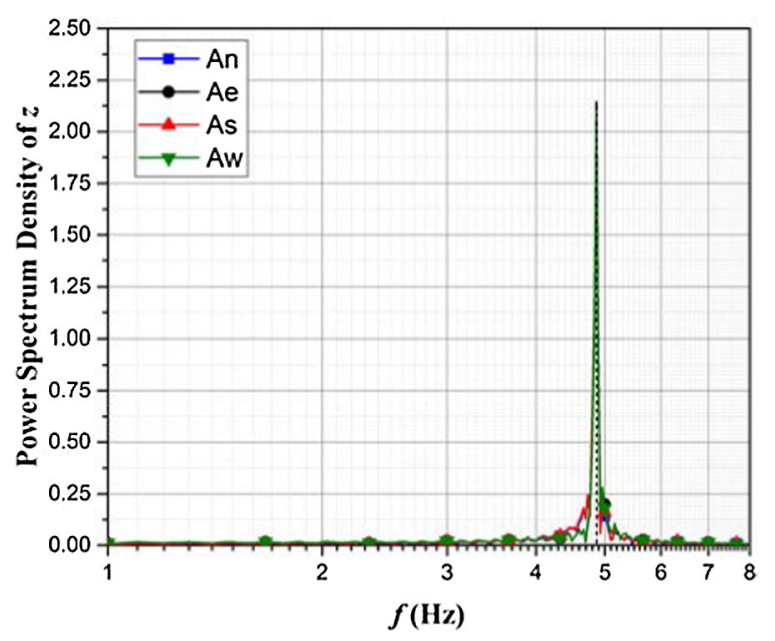

(a)

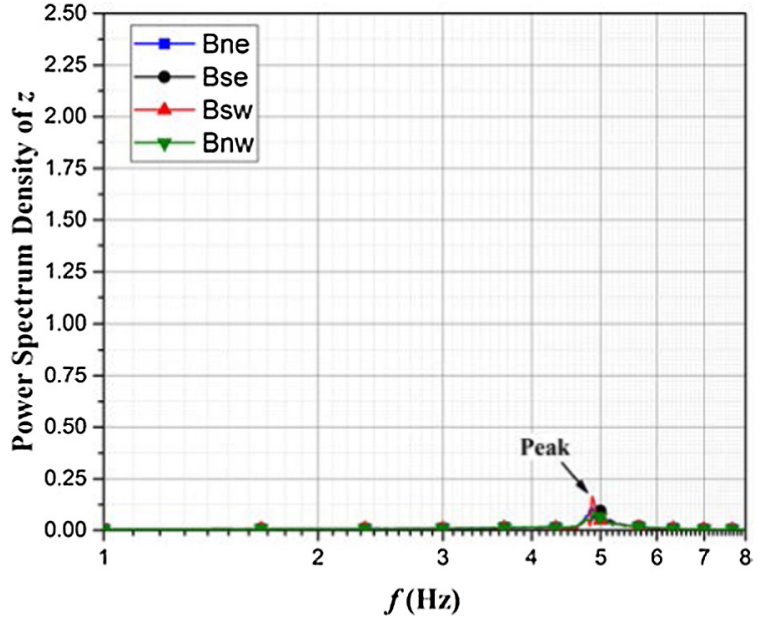

(b)

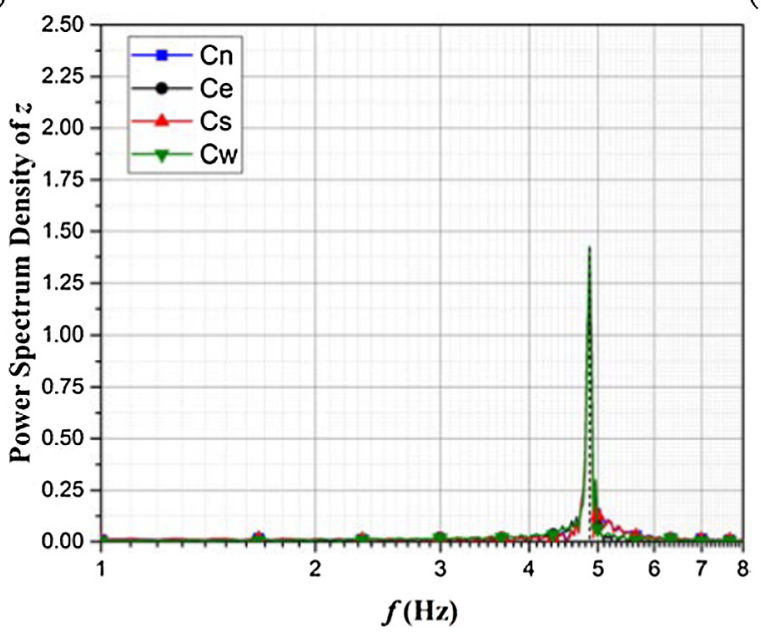

(c)

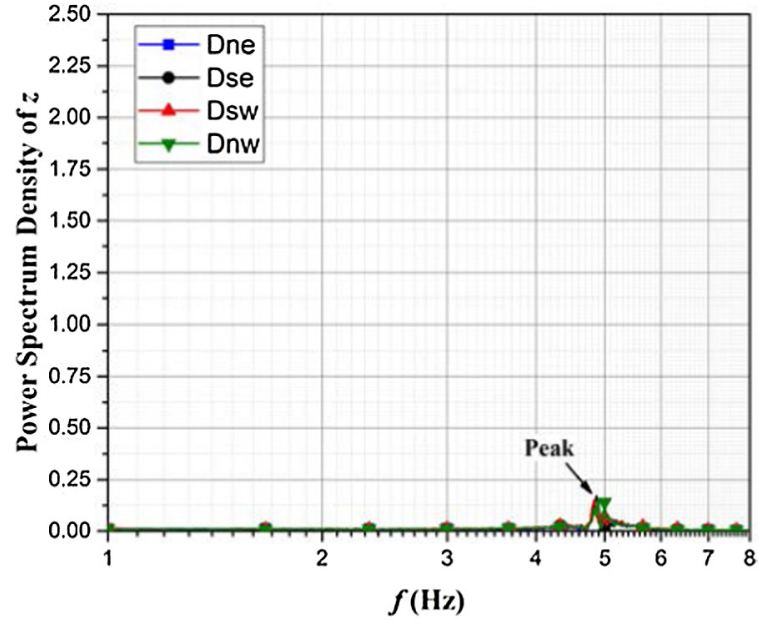

(d)

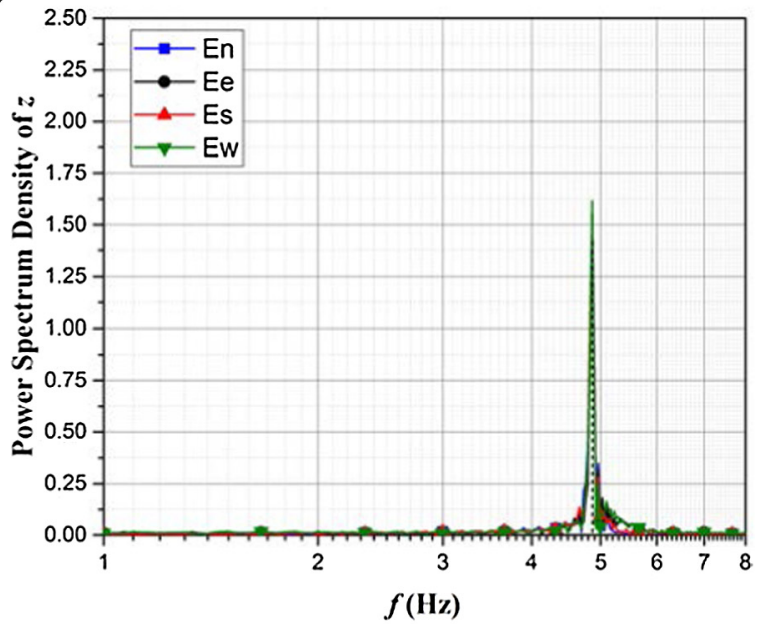

(e)

Fig. 4. Power spectrum density of the displacement signal for FVG case at different row positions (a) row A, (b) row B, (c) row C, (d) row D and (e) row E.

The percentage of relative flow energy associated to each mode can be calculated by:

$\% \epsilon_{i}=\frac{\lambda^{i}}{\sum_{i=1}^{N} \lambda^{i}} \times 100$
In the POD study, each plane contains 1500 snapshots, equally spaced at $0.02 \mathrm{~s}$ apart from each other and each snapshot contains 10000 data points, arranged as $100 \times 100$ points in the radial and tangential directions. The POD strategy is performed and implemented using MATLAB (2010). It is convenient to realize that the 

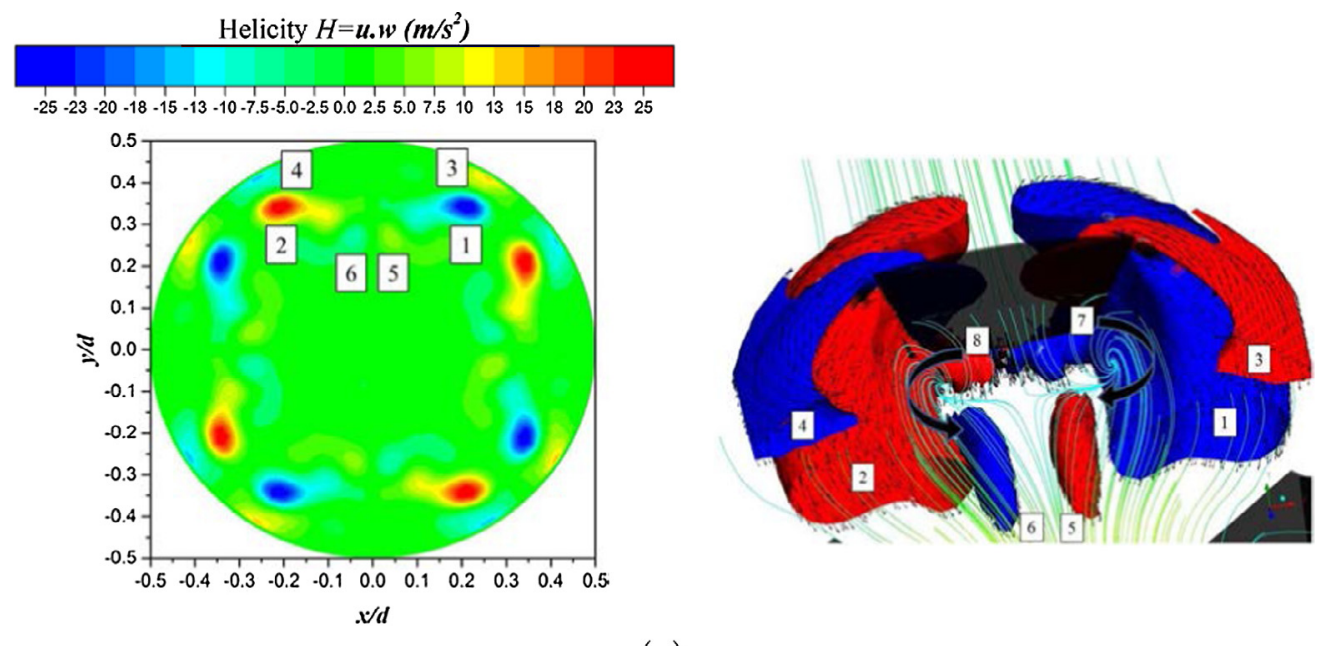

(a)
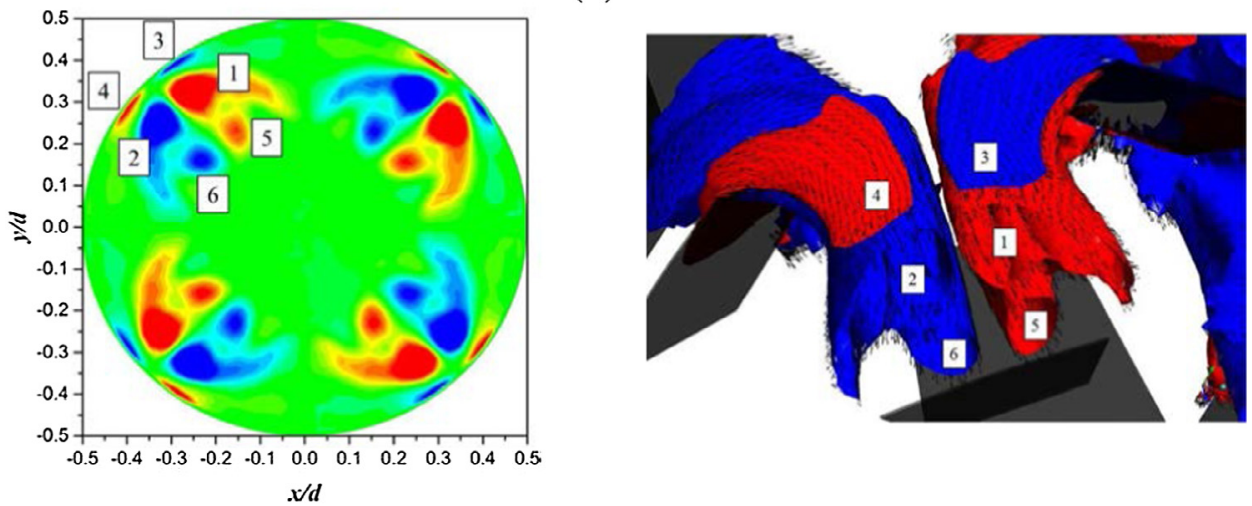

(b)
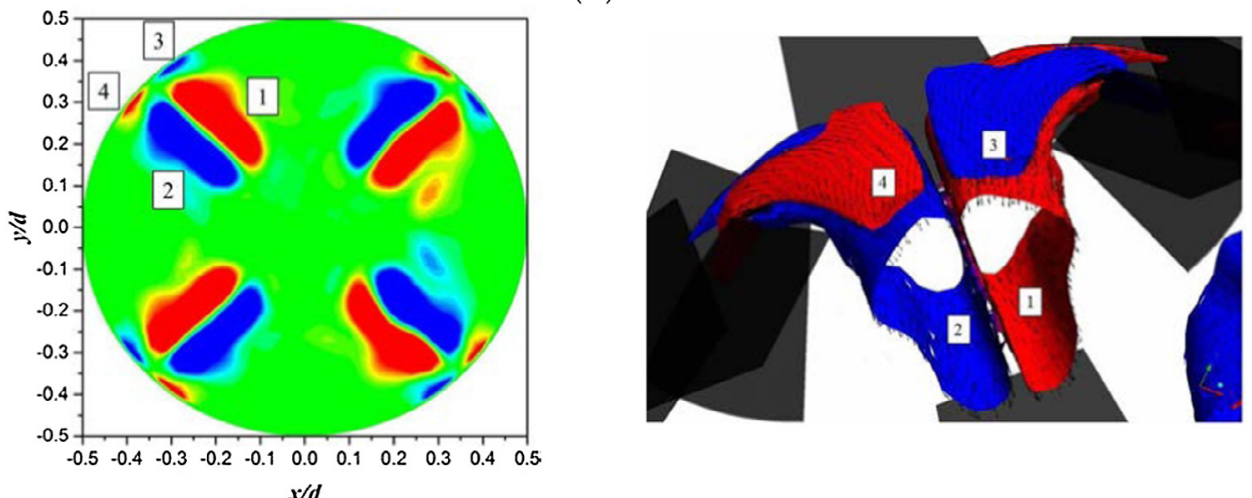

(c)

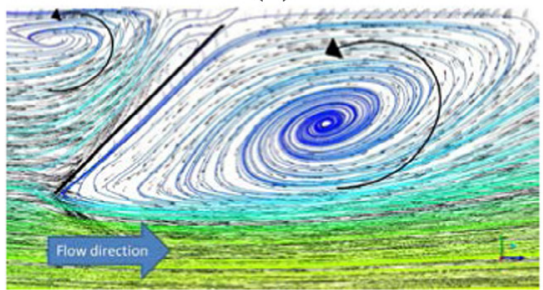

(d)

Fig. 5. Helicity distribution (left side) and 3D view of helicity isoplanes (right side) for the RVG case at row positions, (a) $z / d=1.11$ (downstream row A), (b) $z / d=2.22$ (downstream row $\mathrm{C}$ ) and (c) $z / d=3.33$ (downstream row E); (d) is a side view of tab An.

basis functions do not necessarily reflect a real flow structure in the flow field. Instead, they represent a component of the flow field which can be reconstructed by summing over all the weighted modes.
Table 6 shows the values of the energy content distribution of the first 10 modes. The cumulative energy percentage is defined as $\% \xi_{i}=\sum_{i=1}^{N} \% \epsilon_{i}$. It can be seen that only the first four modes have significant contributions to the total energy in the unsteady flow 
with a total cumulative energy of $88.48 \%$ for plane $1,76.14 \%$ for plane 2 and $64.94 \%$ for plane 3 . Higher eigenvalues have contributions less than $5 \%$ and thus can be neglected. Fig. 6 reveals that the convergence for the $100 \%$ quantity is smaller when indexing from plane downstream of row A (plane 1) to plane downstream of row $\mathrm{C}$ (plane 2) and finally to plane downstream of the last row $\mathrm{E}$ (plane 3 ). This is due to the fact that more complicated and small-scaled flow structures are being produced or convected from past arrays of tabs into the studied one, where the higher eigenmodes contribute more to the total flow energy. Thus, at plane 3 , it is expected to find more small scales and complex flow structures, where higher modes are needed to recover more of the flow energy.

Fig. 7 displays the time history variation during one second of the POD coefficients for the first four modes at the three different planes (i.e. plane 1, plane 2 and plane 3 ). The temporal modes have strong regular shapes and follow periodic cosine and sine functions. From the FFT figures, a peak at $4.86 \mathrm{~Hz}$ is observed which is equal to the frequency of oscillation previously calculated in Section 4.1. This means that the frequency of how the vortices are being created and shed downstream of the tabs is synchronous with the frequency of oscillation and also with the fundamental frequency of the tabs. This phenomenon is identified as lock-in in the literature (Williamson and Govardhan, 2004). Moreover, it can be seen that the POD coefficient variation of the first mode displays small amplitude oscillations, whether the other three modes oscillate with higher amplitudes around zero. This result suggests that if there is a steady component to the flow, then it will be automatically identified as the first mode. In addition, we observe that as the POD index number increases, the temporal behavior of the POD coefficients is degraded, which suggests that the higher POD modes contribute less to the flow energy.

In the subsequent analysis, we present the four modes and the flow structures at plane 3 , since more complex and coherent structures are exhibited and the mechanism of vortex formation coupled with tabs oscillations is nearly the same for all the planes. Fig. 8 illustrates the most energetic four modes which contain information about the vortex formation downstream of the last row $\mathrm{E}$ in the HEV configuration. It can be observed that mode 1 is the most energetic mode with vortices arrangement very similar to the previous RVG case shown in Fig. 5. It is important to note that the counter-rotating vortex pairs in mode 1 are localized near the pipe wall. As we investigate other modes, we can obviously conclude that the vortices are dislocated from their near wall position, and much more assembled at the pipe centerline. In addition, POD modes 3 and 4 represent weaker and higher number of small scale vortices, which represents $12.25 \%$ and $6.14 \%$ of the total flow energy whereas modes 1 and 2 represents $26.88 \%$ and $19.68 \%$ of the total flow energy. Mode 4 in particular can be associated with

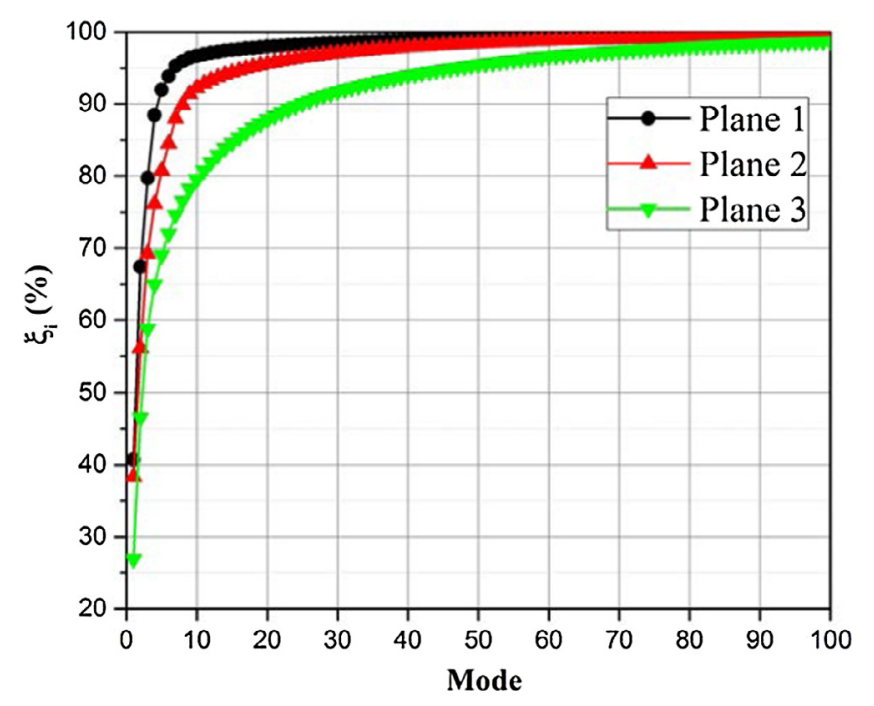

Fig. 6. Cumulative energy content percentage $\% \xi_{i}$ associated with POD modes 1100 using all the 1500 available snapshots for each plane.

the velocity shear layers created at the sides of the tabs as they reach their maximum amplitude positions.

In order to represent a real flow snapshot, POD modes must be multiplied by their POD coefficients. Fig. 9 shows the original helicity field along the reconstructed field for six time instants and during one period of oscillation. Helicity snapshots are first reconstructed using modes 1 and 2 which contribute to $46.56 \%$ of the total flow energy and secondly using all the four dominant modes which contributes to $64.94 \%$ of the total flow energy. Slight differences are present between the original and reconstructed data, which validate the implementation of the "Snapshot POD" algorithm notably, an infinite number of POD modes taken into consideration would be leading to the exact original data. The reconstructed data using the four dominant modes highlight well the signature of the flow structures that contributes the most to the flow energy and neglect the smallest energy structures. The first two eigenmodes represent the near wall vortices plus two pairs of counter rotating vortices located at the center which are either formed by the tabs Ee and Ew or the tabs En and Es as they reach their maximum positions. It is clear that the reconstruction using the eigenmodes 1 and 2 predicts all the near wall vortices, however they fail alone to predict the flow structures and the arrangement of the vortices at Fig. 9(c) and (e). The vortices at these time instants have a similar distribution and structure to mode 3 and mode 4 . By further investigating the reconstructed snapshots using the four modes, we can clearly observe the

Table 6

Relative and cumulative modal energy percentage ( $\% \epsilon_{i}$ and $\% \xi_{i}$ ) of the first 10 modes at different cross sectional planes for the FVG case.

\begin{tabular}{|c|c|c|c|c|c|c|}
\hline \multirow[t]{2}{*}{ Modes } & \multicolumn{2}{|c|}{ Plane 1} & \multicolumn{2}{|c|}{ Plane 2} & \multicolumn{2}{|c|}{ Plane 3} \\
\hline & $\% \epsilon_{i}$ & $\% \xi_{i}$ & $\% \epsilon_{i}$ & $\% \xi_{i}$ & $\% \epsilon_{i}$ & $\% \xi_{i}$ \\
\hline 1 & 40.68 & 40.68 & 38.35 & 38.35 & 26.88 & 26.88 \\
\hline 2 & 26.70 & 67.38 & 17.75 & 56.10 & 19.68 & 46.56 \\
\hline 3 & 12.30 & 79.67 & 13.17 & 69.27 & 12.25 & 58.80 \\
\hline 4 & 8.80 & 88.48 & 6.88 & 76.14 & 6.14 & 64.94 \\
\hline 5 & 3.53 & 92.00 & 4.59 & 80.73 & 4.10 & 69.04 \\
\hline 6 & 1.87 & 93.87 & 3.76 & 84.49 & 2.95 & 71.99 \\
\hline 7 & 1.43 & 95.30 & 3.51 & 88.00 & 2.60 & 74.59 \\
\hline 8 & 0.67 & 95.97 & 1.92 & 89.92 & 1.93 & 76.51 \\
\hline 9 & 0.44 & 96.40 & 1.47 & 91.39 & 1.71 & 78.22 \\
\hline 10 & 0.29 & 96.69 & 0.83 & 92.22 & 1.30 & 79.51 \\
\hline
\end{tabular}




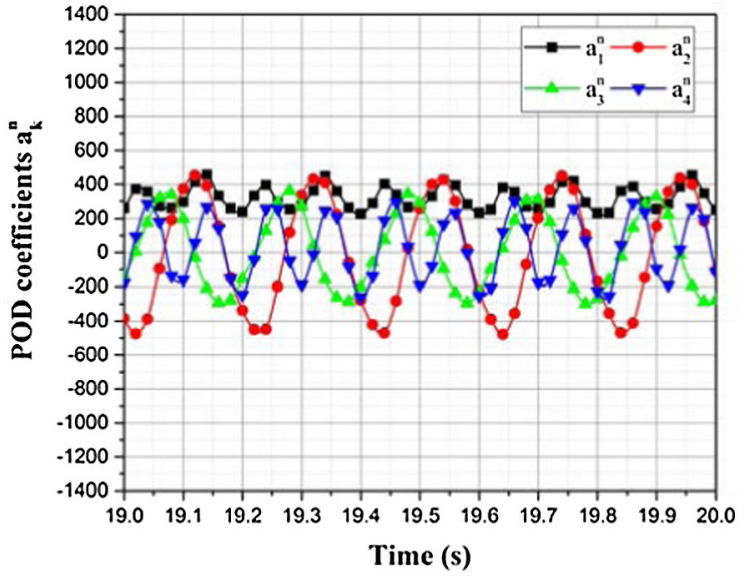

(a)

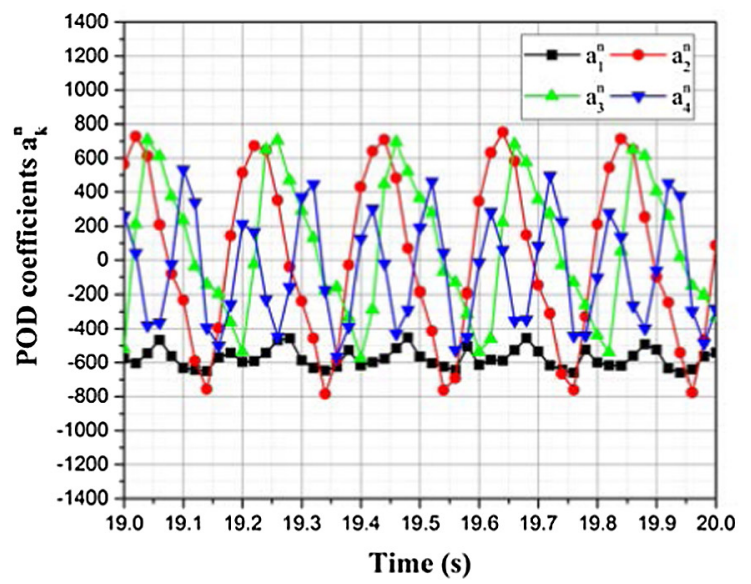

(c)

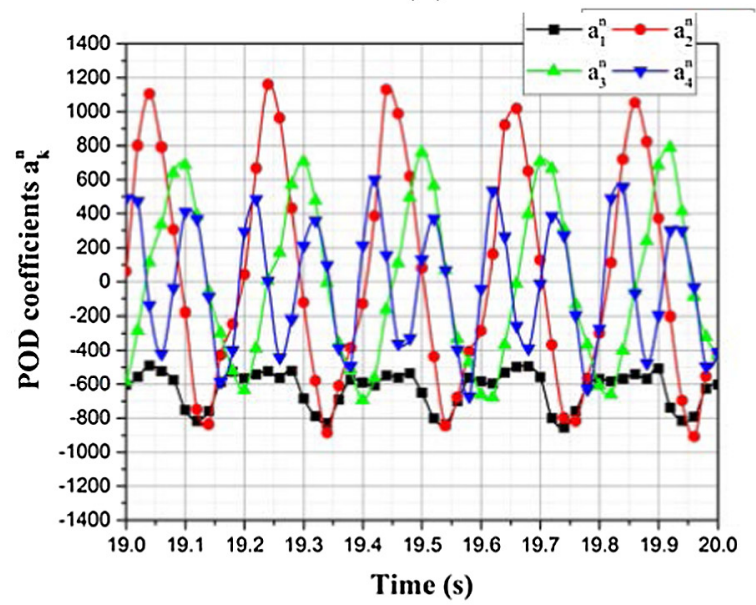

(e)

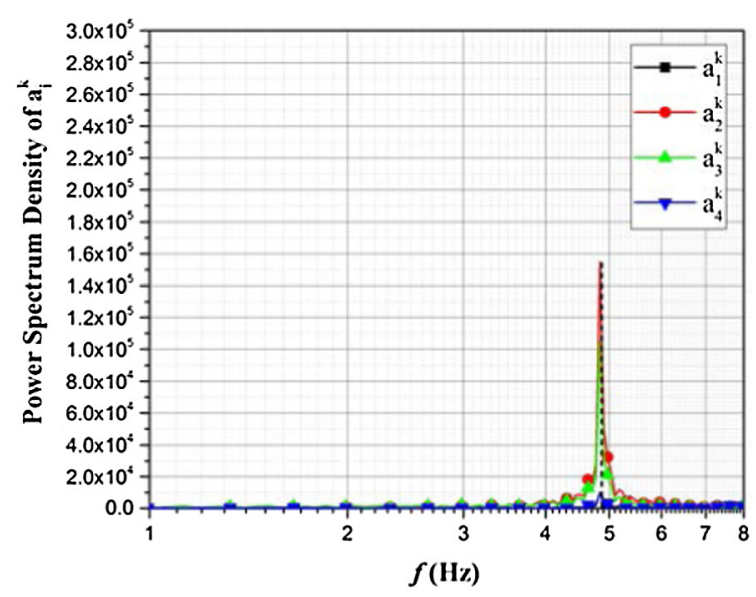

(b)

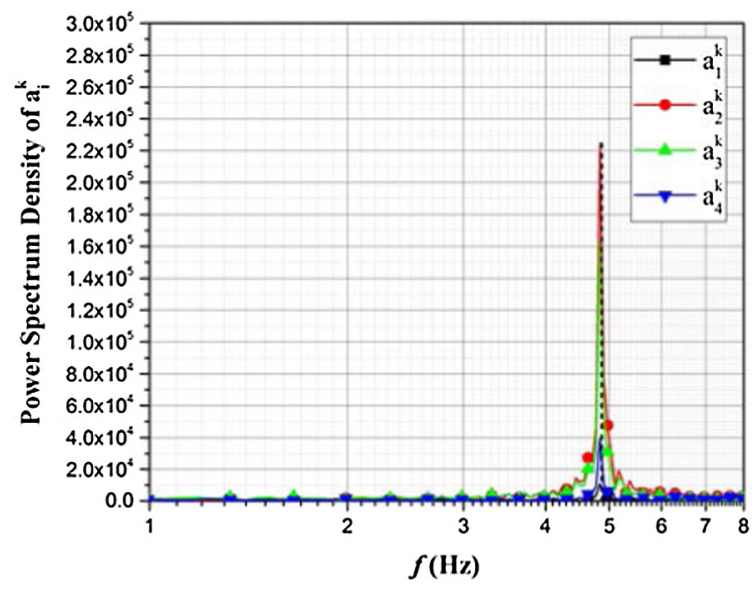

(d)

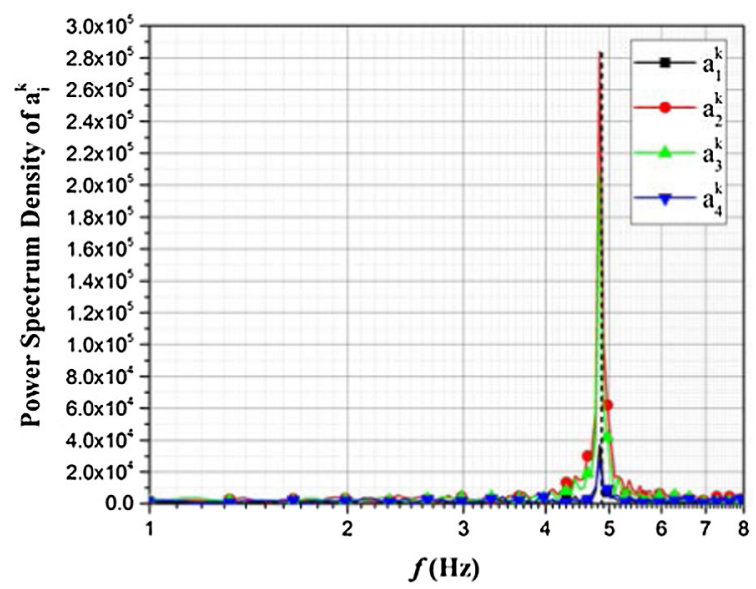

(f)

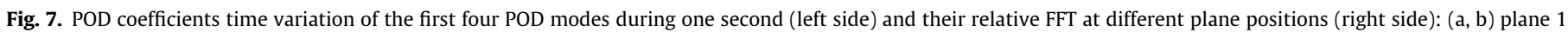
$(z / d=1.11) ;(\mathrm{c}, \mathrm{d})$ plane $2(z / d=2.22)$ and $(\mathrm{e}, \mathrm{f})$ plane $3(z / d=3.33)$.

development of the velocity shear layers along the two sides to the tabs (at index positions 3 and 4 ) as they reach their maximum positions. These two velocity shear layers are actually reduced in size when the tabs deform in the opposite streamwise direction. At this plane, the shear layers disappear as the tabs reach their minimum near wall position as depicted in Fig. 9(c). New formation of the shear layers restarts at Fig. 9(f) where a new cycle of oscillation begins. Moreover, we can notice that the distance between the counter-rotating vortex pairs at index positions 1 and 2 gets smaller as the tabs reach their maximum positions and gets larger at the lower positions, where the tab has a lower impact angle with the flow. This is validated in the 

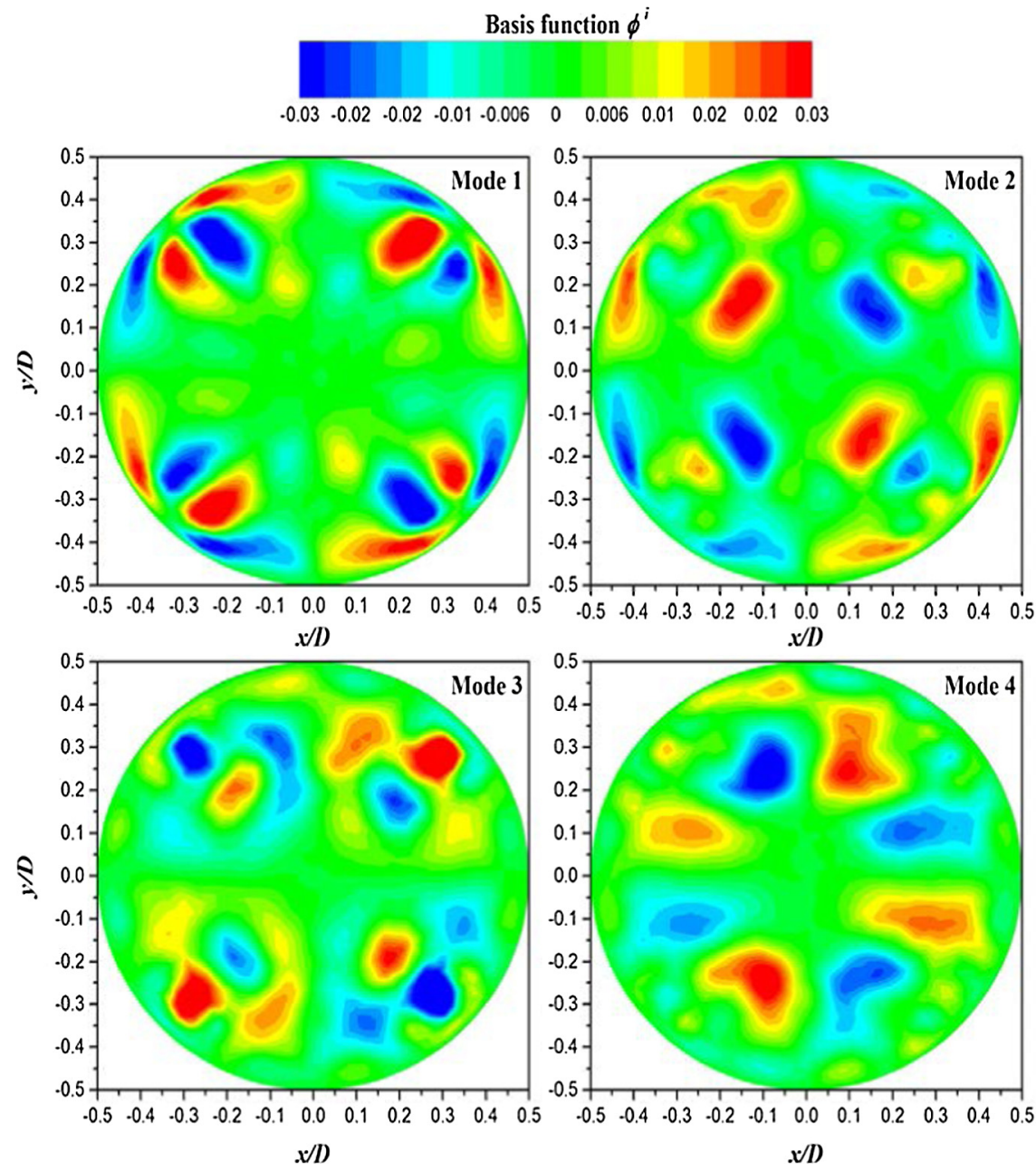

Fig. 8. The first four spatial modes located at plane $3(z / d=3.33)$.

evolution of these vortices in Fig. 9(b), (d) and (f) for tabs Ee and Ew. Similarly, this phenomenon is observed for vortices at index positions 5 and 6.

In fact, looking at the flow structures in the time instants, an important feature is that the vortices are either arranged as packets in the upper and lower part of the domain or in the left and right part of the domain, since there is a phase shifting in the oscillations between tabs Ee and Ew with tabs En and Es. Hence, the tabs oscillations are associated with the phenomenon of creation, destruction and dislocation of the flow structures. This is found to be in contrast with the RVG case, where all flow structures are found near the wall and are not dislocated from their positions. Fig. 10 shows the isoplanes of helicity at tab En during one period of oscillation. At Fig. 10(a), the tab is at its highest amplitude position. We can observe the velocity shear layer wrapping around the tab sides and being deflected towards the low pressure area in the wake region. As the tabs oscillate in the negative $z$-direction, these two shear layers change in shape and are more stretched horizontally than vertically since the deformation of the tabs reduces the contact of the flow with the two tabs sides. The velocity shear layers disappear at Fig. 10(d). At the minimum tabs position and since the incoming flow experiences a strong adverse streamwise pressure gradient coupled with the cross-stream gradient generated by the curvature of the flow around the tab, two large scale counter-rotating vortices are formed. These vortices resemble to the horseshoe vortex-like structure (Dong and Meng, 2004) which wraps around the tab in the region of the bottom boundary layer and extends with their two arms to the sides of the wake.

\section{Heat transfer performances}

In this section, the temperature distribution comparison between the FVG and RVG cases are discussed, followed by quantification of the heat transfer performance using the parameters of Nusselt number $N u_{z}$ and thermal performance factor $\eta$.

Fig. 11 shows a side cross sectional view of the temperature distribution contours for the RVG case. It can be observed that the thermal boundary layer is renewed after each passage of tabs rows. However, overheated regions are observable directly in the wake region behind the tabs. This can be explained by the streamwise velocity streamlines shown in Fig. 12 where recirculation regions are found behind the tabs. In these regions, heat stays trapped in the stagnant transverse vortices and can only be transported to the core region by diffusion. These recirculation regions are known to have a negative impact on heat transfer performance.

Fig. 13 plots a side cross sectional view of the temperature distribution contours for the FVG case during one period of oscillation. By comparing Figs. 11 and 13, the presence of FVG displays clearly the impact of tabs oscillation on the wake region and the reduction of the thermal boundary layer and hence the elimination of the overheated regions behind the flexible tabs. Fig. 14 shows the streamline velocity $U_{z}$ distribution for the FVG case during one period of 

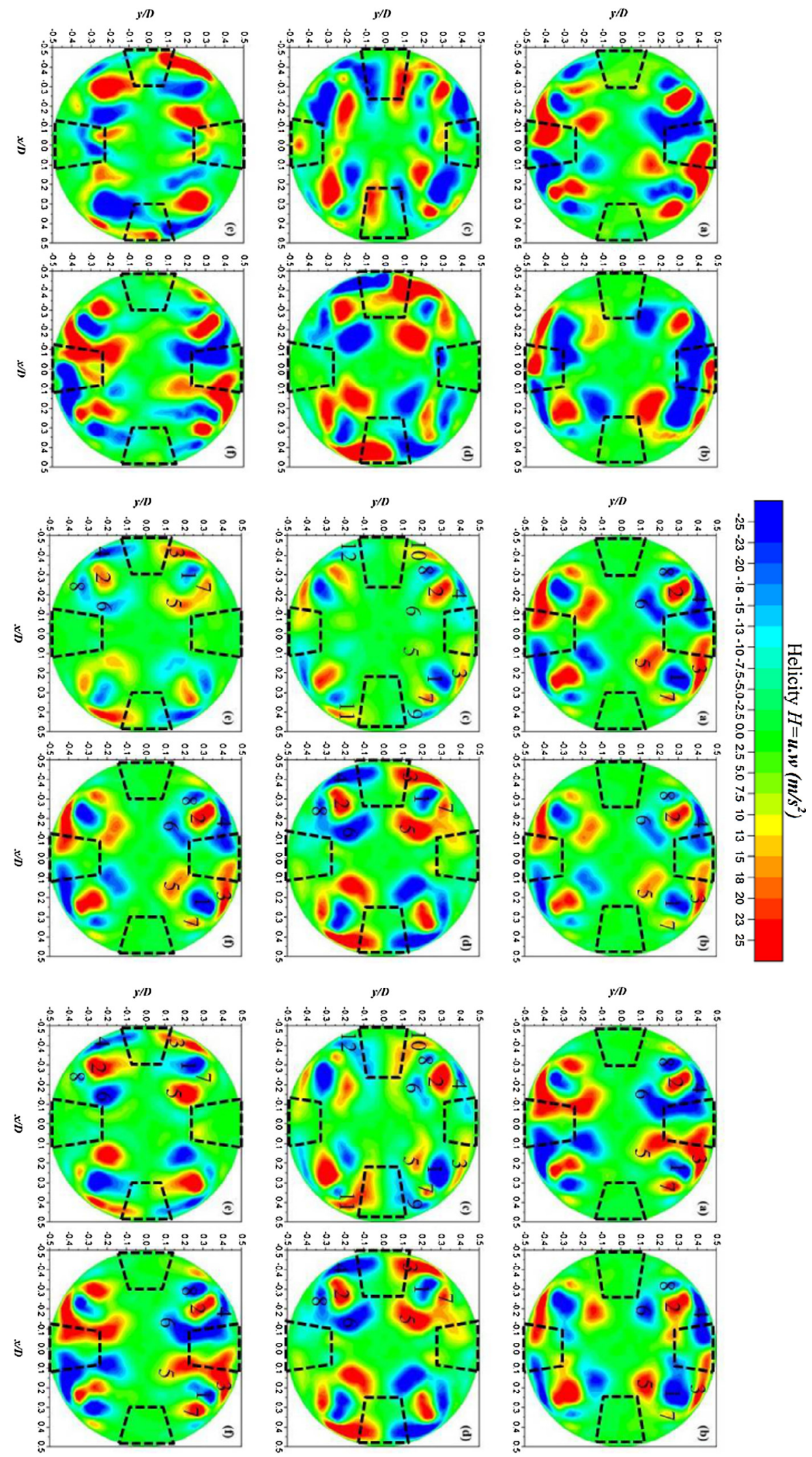

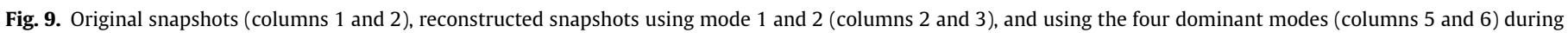

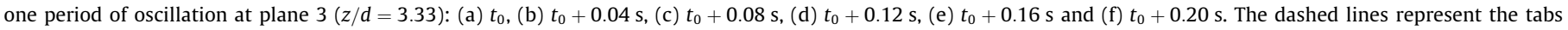
deformation positions.

oscillation. By looking at Fig. 14 and the zoomed-in region in Fig. 15, we can see the unsteadiness of the flow behind the flexible tabs. In fact as a tab oscillates in the streamwise direction, a transverse vortex is formed which scales up to the tab length as seen in Fig. 15. The transverse vortices are periodically shed due to the shear layer instability generated at the tip of each tab as stored 


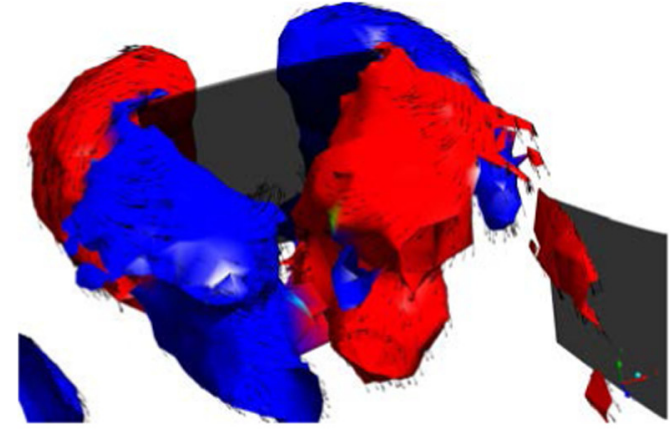

(a)

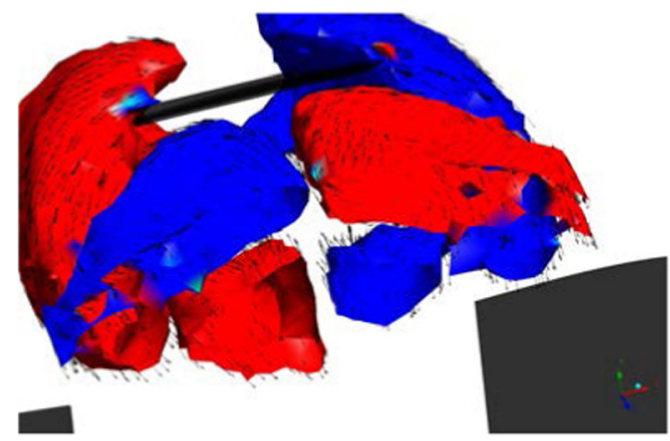

(c)

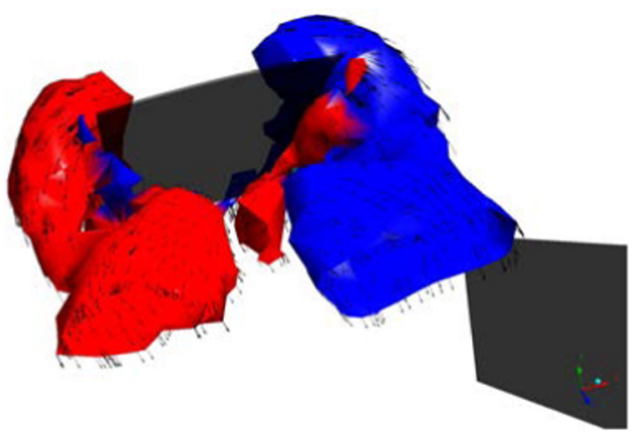

(e)

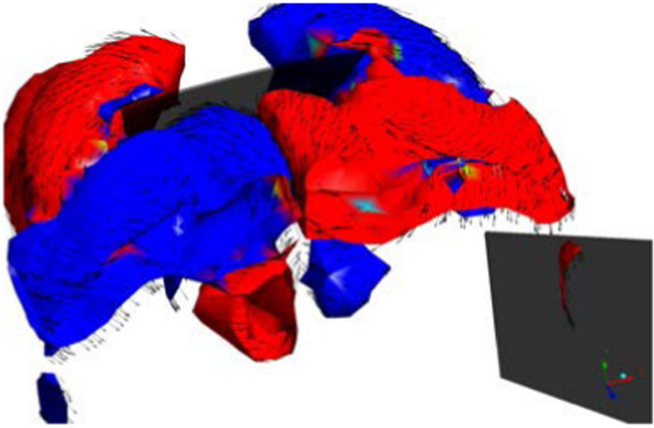

(b)

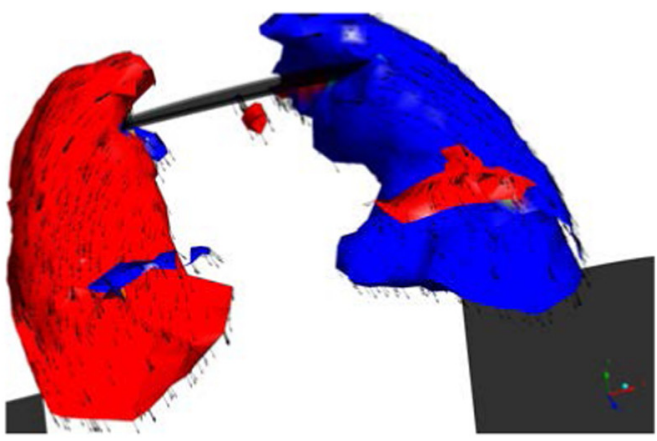

(d)

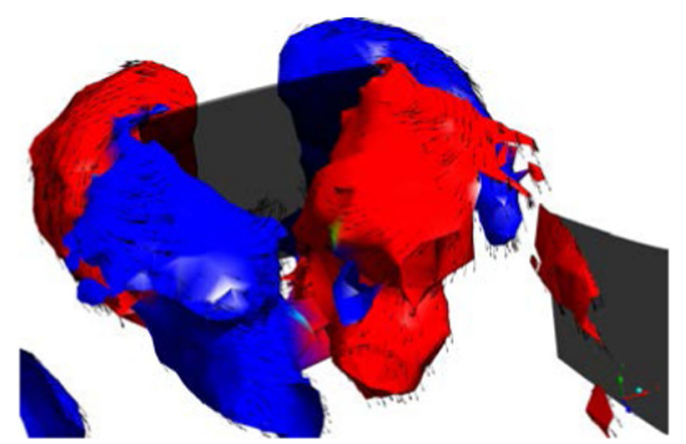

(f)

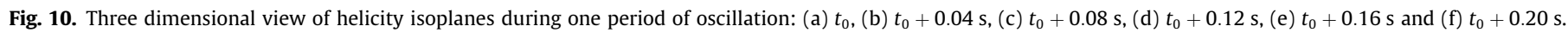

kinetic energy is released and it then oscillates in the opposite streamwise direction. These vortices are convected downstream instead of being stationary like in the RVG case. This unsteadiness plays a crucial role in transporting the thermal energy from the wall to the core of the fluid or inversely from the core of the fluid to the wall.

In addition, by comparing Figs. 11 and 13, we can notice several important differences. In Fig. 11, in the wake region downstream the last row $\mathrm{E}$, the recirculation region extends from $z / d=3$ to approximately $z / d=4$ until the flow gets further deviated to a cone expanding shape reaching the wall boundaries after $z / d=5$ and thus increasing the temperature gradients after this position. In contrast to the preceding observation, Fig. 13 illustrates the reduction of the thermal boundary layer behind the tabs from $z / d=3$ to $z / d=4$ for the FVG case. However, after $z / d=4$, the RVG case displays higher temperature gradients near the pipe wall and an important reduction in the thermal boundary layer. In fact, because of the high streamwise deformation of the tabs of row $E$, the fluid experiences a jet-like flow which is mostly located at the pipe centerline and in partial contact with the wall. This effect deteriorates the heat transfer performance after this position for the FVG case.

In order to quantify the heat transfer process, we calculate the time-averaged local Nusselt number $\overline{N u}(z)$ along the pipe length by:

$\overline{N u}(z)=\frac{1}{t_{2}-t_{1}} \int_{t_{1}}^{t_{2}} \frac{q_{w}^{\prime \prime}(z, t) d}{k_{t h}\left(T_{w}-T_{b}(z, t)\right)} d t$

where $t_{1}$ is the time step at which periodic high amplitude oscillations are reached $\left(t_{1}=19 \mathrm{~s}\right)$ and $t_{2}$ is the final instant in seconds.

Fig. 16 shows the local Nusselt number for the FVG, RVG and empty pipe simulations. It can be seen that the evolution of the local Nusselt number is spatially periodic due to the presence of equally spaced arrays of tabs for both the FVG and RVG cases. At each VG location, the Nusselt number starts to increase from the leading edge of the tab due to the generation of vortices and then progressively decreases downstream. The effect of transverse vor- 


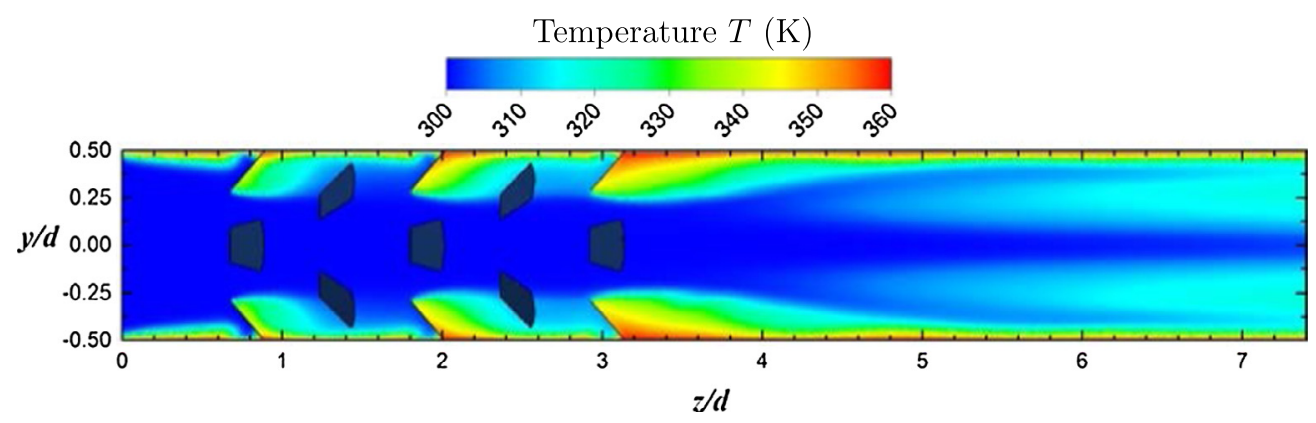

Fig. 11. Temperature distribution for the RVG case in the tab symmetry plane $y z$.

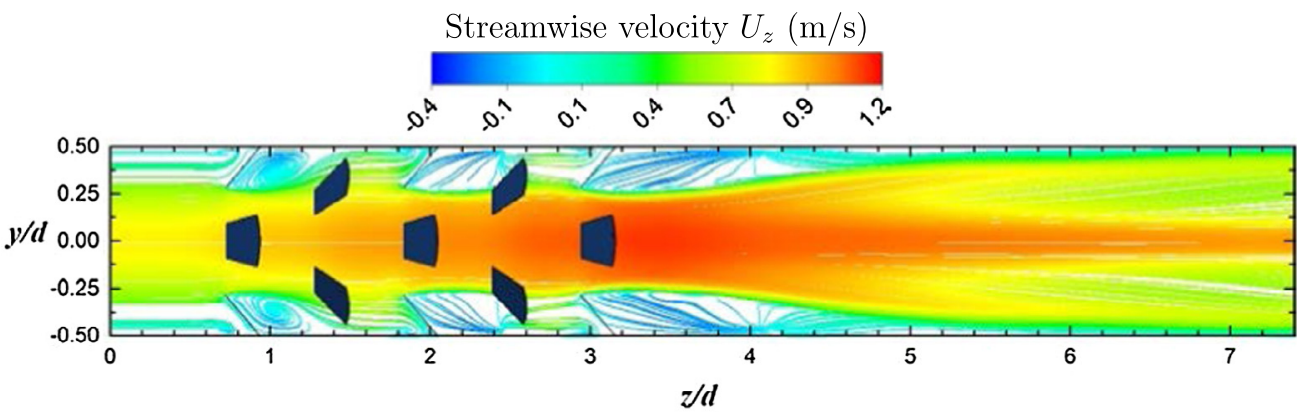

(a)

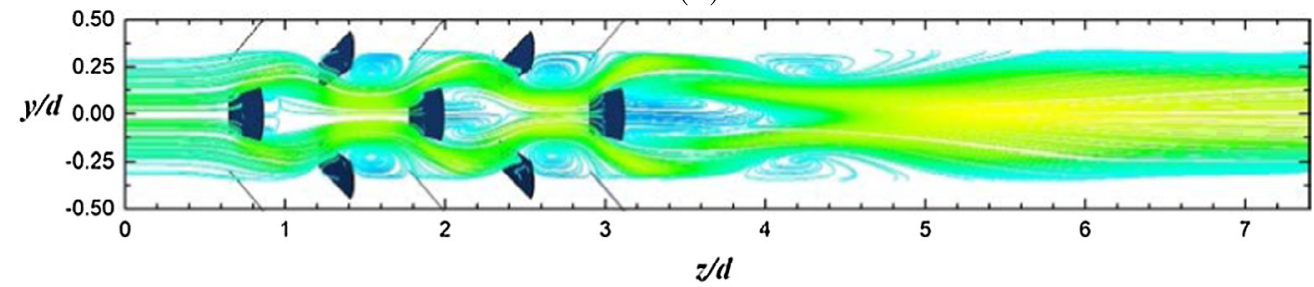

(b)

Fig. 12. Streamwise velocity streamlines for the RVG case: (a) in the symmetry plane $y z$ and (b) in the plane $x z$ at position $y / d=0.37$.

tex shedding in the FVG case and the trapped flow remaining in the recirculation region in the RVG case previously mentioned is reflected in the local Nusselt number values. In fact, the FVG case displays higher values of Nusselt number than the RVG case at the wake region of every row i.e. after every peak of Nusselt number. Specifically, in the wake region of the last row $(z / d=3$ to $z / d=4)$, the Nusselt number is significantly higher in the FVG than in the RVG case, where it keeps decreasing at distances larger than $z / d=4$. However, the Nusselt number in the RVG starts to increase at position $z / d=4$, which is directly correlated to the deviation of the flow to the wall boundaries and elimination of the thermal boundary layer as seen in Fig. 11. The intensification of the spatially averaged local Nusselt number $\left(\frac{1}{L} \int_{0}^{L} N u_{z} d z\right)$ with respect to the empty pipe flow is about $112 \%$ for the FVG case and $105 \%$ for the RVG case.

Nevertheless, another difference observed between the RVG and FVG cases from the plots of local Nusselt number in Fig. 16 is that the peaks of $N u_{z}$ for the RVG case are surprisingly higher than the FVG case at positions where the tabs undergo high amplitude oscillations (row A $(z / d=0.83)$, row $C(z / d=1.96)$ and row $\mathrm{E}$ $(z / d=3.07))$. Moreover, at the positions where the flaps have negligible oscillation amplitudes, the peaks for the FVG case are higher than that of the RVG case (row B $(z / d=1.40)$, row $\mathrm{D}(z / d=2.52)$.
To better explain the discrepancies of the peak local Nusselt between both cases and the reasons why the FVG case displays higher $N u_{z}$ peaks at low oscillation amplitudes and lower $N u_{z}$ peaks at higher oscillation amplitudes, we investigated the behavior of flow and heat transfer performances at rows $\mathrm{C}$ and $\mathrm{D}\left(\mathrm{Nu}_{z}\right.$ peaks at $z / d=1.96$ and $z / d=2.52$ ).

Fig. 17(a) and (b) shows the streamwise velocity at the center of the pipe along the $x$ and $y$ directions at position $z / d=1.96$ where the tabs undergo high amplitude oscillation, whereas Fig. 17(c) and (d) are considered at positions $z / d=2.52$ and associated with small amplitude oscillation. We can observe from Fig. 17(a) and (b) that the streamwise velocity for the FVG case is higher than the RVG case at the center due to the increase in the blockage area as the tabs deform to their streamwise positions. However, the velocity profile for the FVG case is narrower than the RVG case as the distance increases further from the center of the pipe. This is validated by the velocity contours of Fig. 14(c) as we can observe at position $z / d=1.96$ the flow experiencing a high speed at the narrower neck area and a low speed at the wake of the tabs. The high speed flow impinges the next row $\mathrm{D}$ at position $z / d=2.52$. We can clearly see the difference in the RVG case from Fig. 12 where the flow have a wider neck region at $z / d=1.96$ (row C) with lower maximum velocity at the center than the FVG case. 

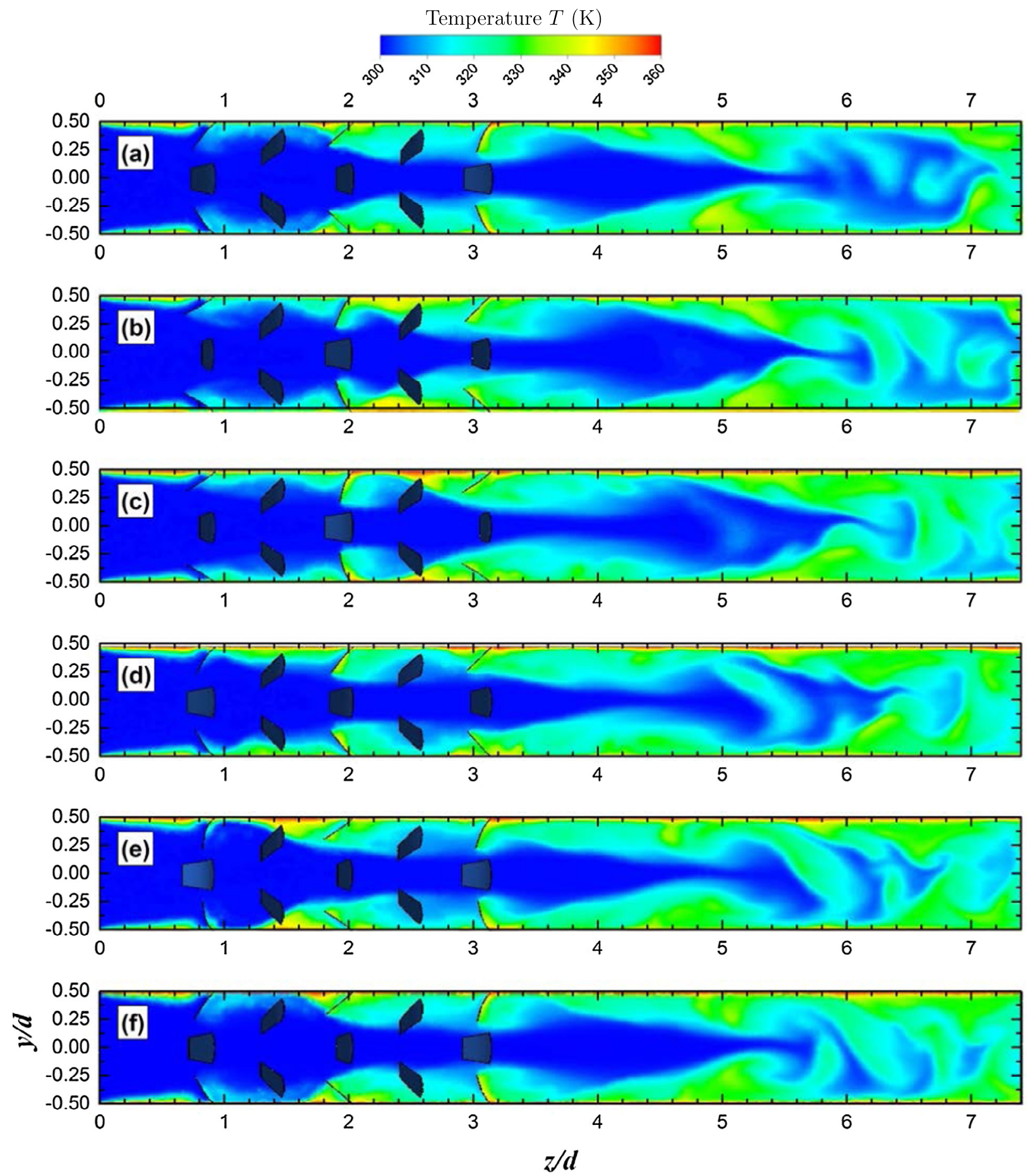

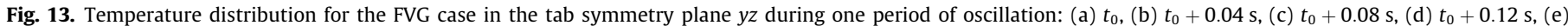
$t_{0}+0.16 \mathrm{~s}$ and (f) $t_{0}+0.20 \mathrm{~s}$.

Consequently, the flow impinges the next row $\mathrm{D}$ at position $z / d=2.52$ at lower speed specifically at the wall since row $\mathrm{D}$ is tangentially rotated by an angle of $45^{\circ}$. This is supported by the velocity profile of Fig. 17(c) and (d) where we can see the higher velocity distribution for the FVG case at positions $y / d<-0.3$ and $y / d>0.3$ or $x / d<-0.3$ and $x / d>0.3$. The increase in the velocity gradients near the wall further increases the convective heat transfer and induces an improved thermal effect.
The global Nusselt numbers $N u_{0-L}$ calculated for the empty pipe, RVG and FVG cases are next summarized in Table 7. The temporal mean of the FVG case is performed from an initial time $t_{i}=19 \mathrm{~s}$ till the final time $t_{f}=30 \mathrm{~s}$. The overall heat transfer enhancement with respect to the empty pipe is about $118 \%$ for the FVG case and about $97 \%$ for the RVG case, which is a good indicator about the ability of the free flexible oscillation in enhancing heat transfer without relying on external additional source of energy. However, 

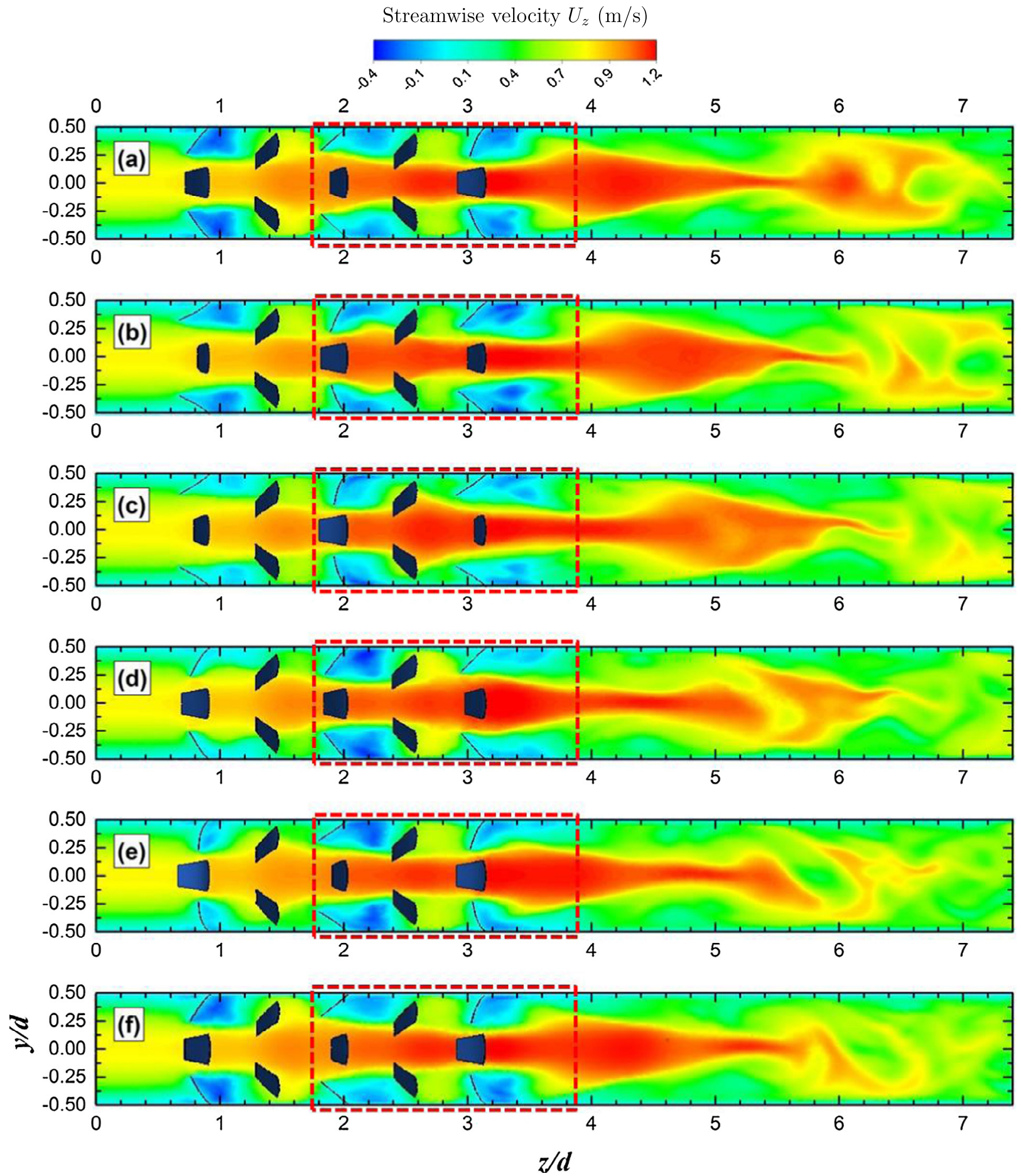

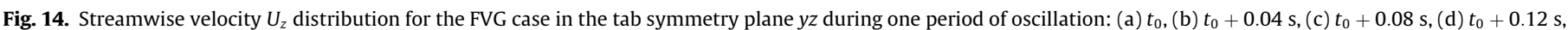
(e) $t_{0}+0.16 \mathrm{~s}$ and (f) $t_{0}+0.20 \mathrm{~s}$. The dotted region is zoomed in and shown in Fig. 15 .

in order to compare the thermal efficiency of the RVG and the FVG cases, the thermal performance factor $\eta$ (Promvonge and Thianpong, 2008) is further investigated. It is defined as the ratio of convective heat transfer in the studied geometry to that in a straight pipe flow and can be written as:

$\eta=\left(\frac{N u_{(0-L)}}{N u_{(0-L), 0}}\right)\left(\frac{f}{f_{0}}\right)^{-\frac{1}{3}}$ where the 0 subscript stands for the empty pipe results. The RVG case result is $\eta=0.995$ while the FVG case result is approximately similar $\eta=0.987$. Although the FVG case increases the overall heat transfer, it also induces a much higher penalty of pressure drop than the RVG case. The tabs motion absorbs the flow kinetic energy and at some time instants increases the pipe blockage ratio when reaching their maximum streamwise position. A further optimization should be thus done on the tabs mechanical properties, angle 


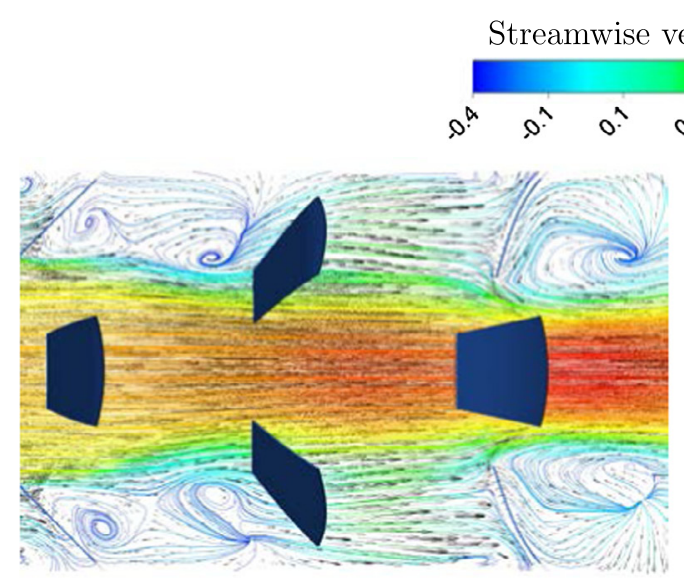

(a)

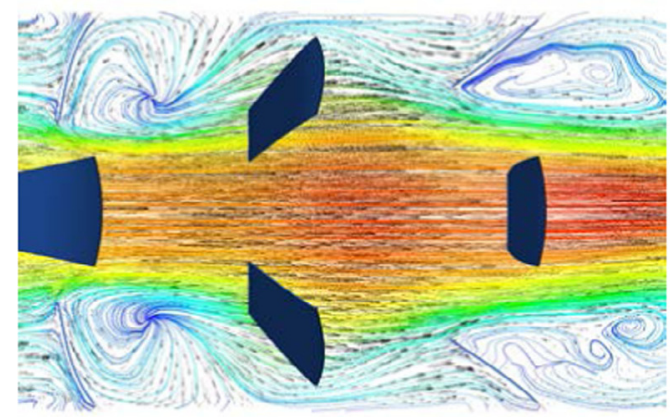

(c)

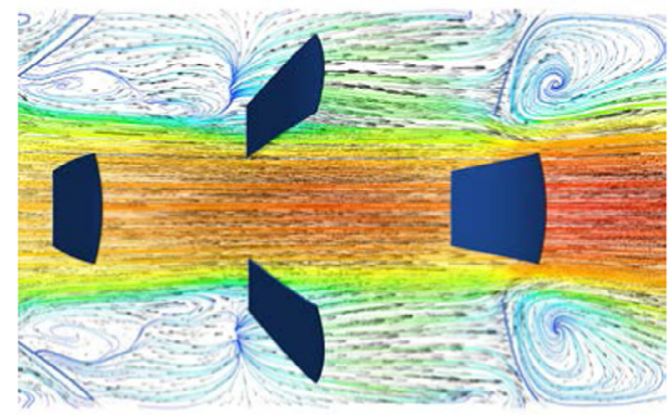

(e)

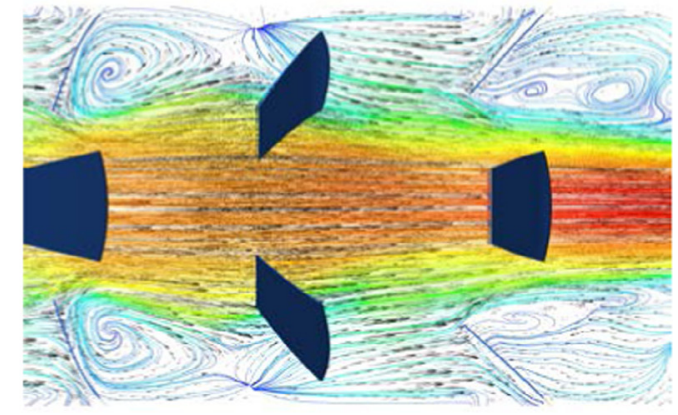

(b)

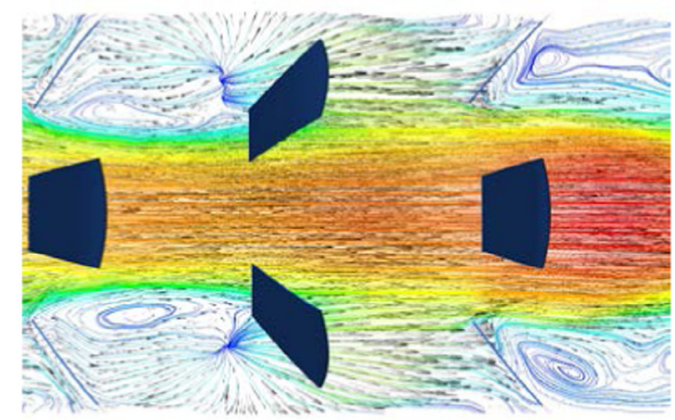

(d)

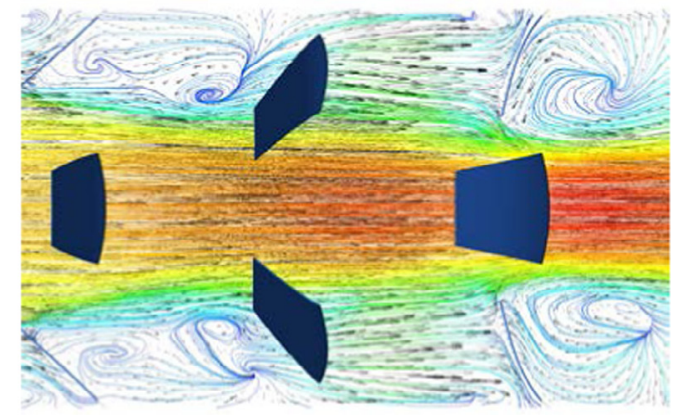

(f)

Fig. 15. The zoomed in streamwise velocity distribution of the dotted region in Fig. 14 .

of attack or arrangement in order to intensify their thermal performance and reduce the pressure drop.

\section{Mixing analysis}

In this section, the mixing performance is compared between the two FVG and RVG cases. As shown previously in Fig. 1, the scalar field is initially divided into a left half $c=0$ and a right half $c=1$ and then mixes with the flow instability. The mixing performance is evaluated for both cases by computing the mixing index (MI) of the passive scalar $c$.

Fig. 18 shows the scalar distribution downstream the last row $\mathrm{E}$ at a position $z / d=3.33$ for the RVG case. It is observed that the tabs Ee and Ew have small or even negligible effect on mixing since the vortices formed by these tabs are localized in the right and left planes and no scalar exchange is occurring between the two halves. However the mixing is more clearly observed at the north and south positions where the vortices are formed at the wake downstream these tabs and near the separation zone of the two different scalar halves. The role of these vortices is thus to engulf one scalar into another.

Fig. 19 displays the scalar distribution for the FVG case at a position $z / d=3.33$ during four time instants. Here the mixed zone is much larger at the center since the tab oscillation dislocates the vortices to the center of the tube allowing a greater exchange of different scalar concentration, as stated before in Section 4.2. The mixing layer is propagated from the north side to the south side and varies in thickness as the tab change in position. This thickness is much likely influenced by the position of tabs Ee and Ew. In fact, as the tabs Ee and Ew reach a high streamwise amplitude position, an area of negative pressure in the wake of these tabs is created. As a result, the flow rushes from the center to the negative area region, stretching along the mixing layer presented at the center of the pipe as in Fig. $19\left(t_{3}\right.$ and $\left.t_{4}\right)$. Moreover, at the north and south positions and at the tab left and right sides, we can observe 


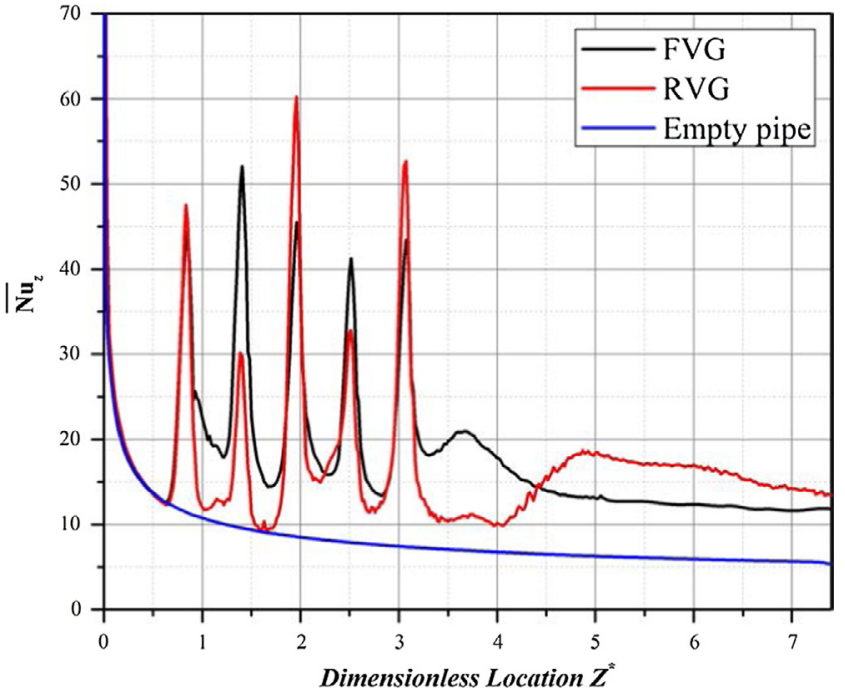

Fig. 16. Time averaged local Nusselt number $\overline{N u}_{z}$ of the FVG and RVG cases with respect to the dimensionless location $Z^{*}$.
Table 7

Statistical information about the global Nusselt number $\mathrm{Nu}_{0-L}$ computed at the outlet of the pipe for the different cases.

\begin{tabular}{cccc}
\hline$N u_{0-L}$ & FVG & RVG & Empty pipe \\
\hline Mean & 18.061 & 16.394 & 8.301
\end{tabular}

that as the tabs oscillate in the opposite streamwise direction, the region of well mixed scalar expands as shown by the vectors drawn in Fig. 19. This is explained by the fact that as the tabs oscillate in the opposite streamwise direction, the horseshoe-like vortex wraps around the tab by a much wider turn since the adverse and cross pressure gradient are higher in the opposite streamwise motion. This phenomenon is furthermore illustrated in Fig. 20, which shows the streamlines around tab En at two positions: (a) maximum positions where the flow wraps the side of the tabs and enters the negative pressure region directly and (b) where the flow takes a much wider wrap, turns around the two sides of the tabs and gets responsible for the effect seen in the expanding vector drawn in Fig. 19.

To quantitatively analyze the mixing performance, we calculate the mixing index as in Lambert and Rangel (2010) to assess the

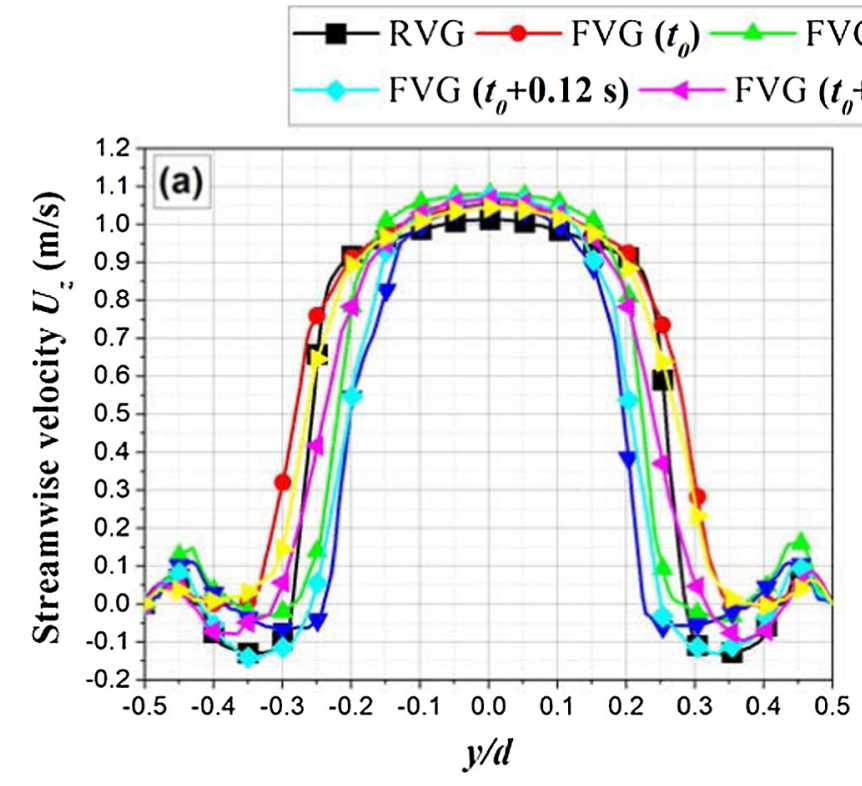

$$
\begin{array}{cc}
\mathrm{FVG}\left(\boldsymbol{t}_{0}+\mathbf{0 . 0 4} \mathrm{s}\right) \longrightarrow \mathrm{FVG}\left(\boldsymbol{t}_{0}+\mathbf{0 . 0 8 \mathrm { s }}\right) \\
\mathrm{FVG}\left(t_{0}+\mathbf{0 . 2 0 \mathrm { s }}\right)
\end{array}
$$
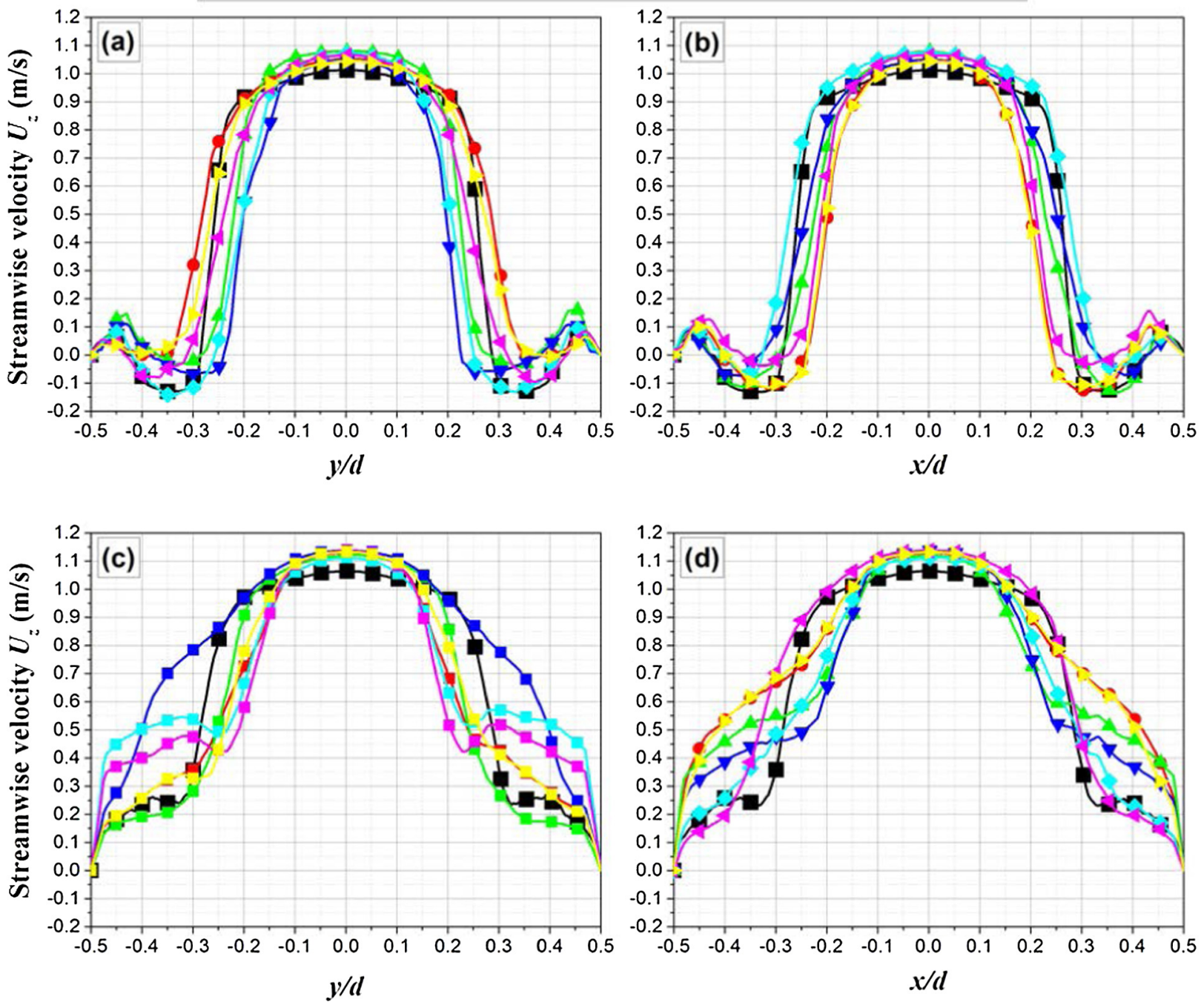

Fig. 17. Streamwise velocity distribution along $x$ and $y$ directions at two different positions: (a, b) at $z / d=1.96$ and (c, d) at $z / d=2.52$. 


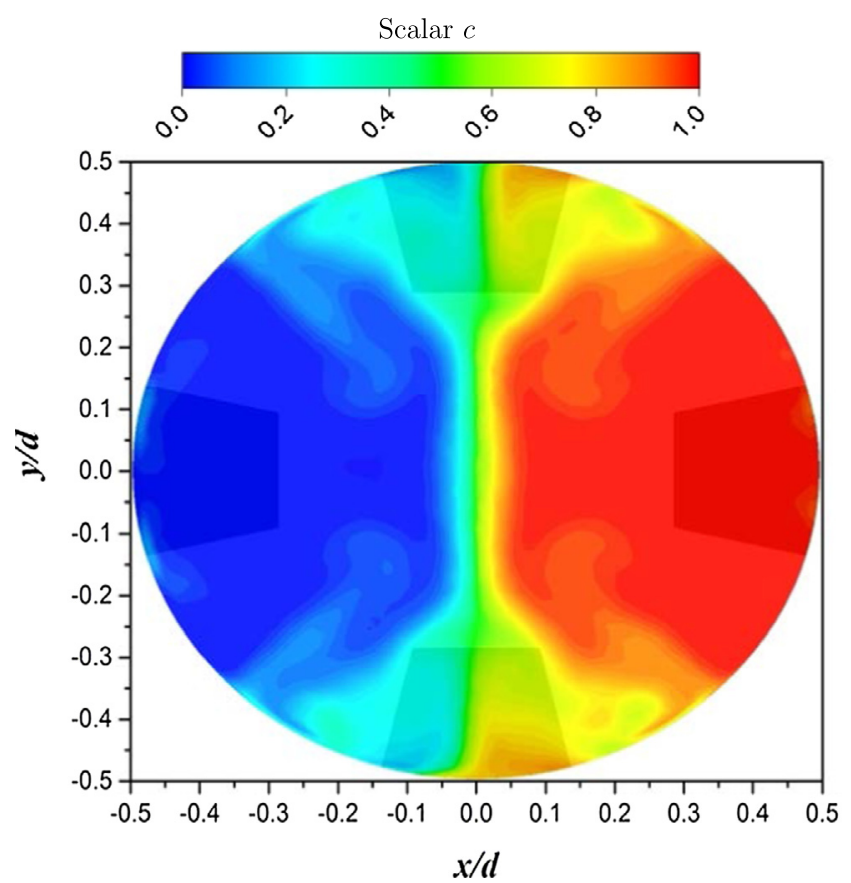

Fig. 18. Scalar field distribution at a cross sectional view $(z / d=3.33)$ for the RVG case. quality of the mixture observed in the RVG and FVG cases. Here, the mixing efficiency along all the pipe length is computed using the time-averaged mixing index at different positions in the pipe:

$$
\begin{aligned}
\overline{\mathrm{MI}}_{c}(z)= & \frac{1}{t_{2}-t_{1}} \\
& \times \int_{t_{1}}^{t_{2}}\left[1-\frac{1}{c_{\text {ave }}(z, t)}\left[\frac{\sum_{j=1}^{n}\left(c_{j}(x, y, z, t)-c_{\text {ave }}(z, t)\right)^{2}}{n}\right]^{1 / 2}\right] d t
\end{aligned}
$$

where $c_{\text {ave }}(z, t)$ is the averaged value of $c$ at a given position $z$ and at time $t . n$ is the number of cells at a cross sectional position, i.e. the number of samples where the concentration value is extracted. Calculation is made from $t_{1}=19 \mathrm{~s}$ to $t_{2}=30 \mathrm{~s}$ to only include the quasi periodic oscillation regime. Consequently, for no mixture case, $\mathrm{MI}=0$ and for fully mixed case, $\mathrm{MI}=1$.

Fig. 21 shows the local mixing index spatially varying for the FVG and RVG cases. It is clearly seen that the tabs oscillation observable in the FVG case enhances the mixture quality better than in the RVG configuration since unsteady vortices are formed and are then dislocated to the center, acting as internal agitators that mix the left with the right scalar zones. All along pipe length, the FVG configuration displays better mixing performance than the RVG case. In practical mixers, this will consequently lead to shorter mixing length and more compact equipments. The mixture quality at the outlet of the pipe reached finally 0.718 for the FVG case

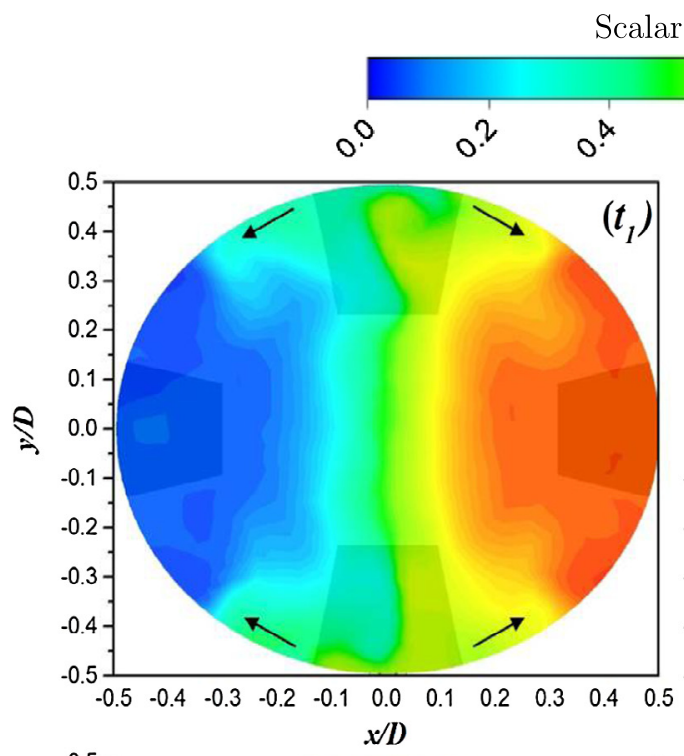

Scalar $c$
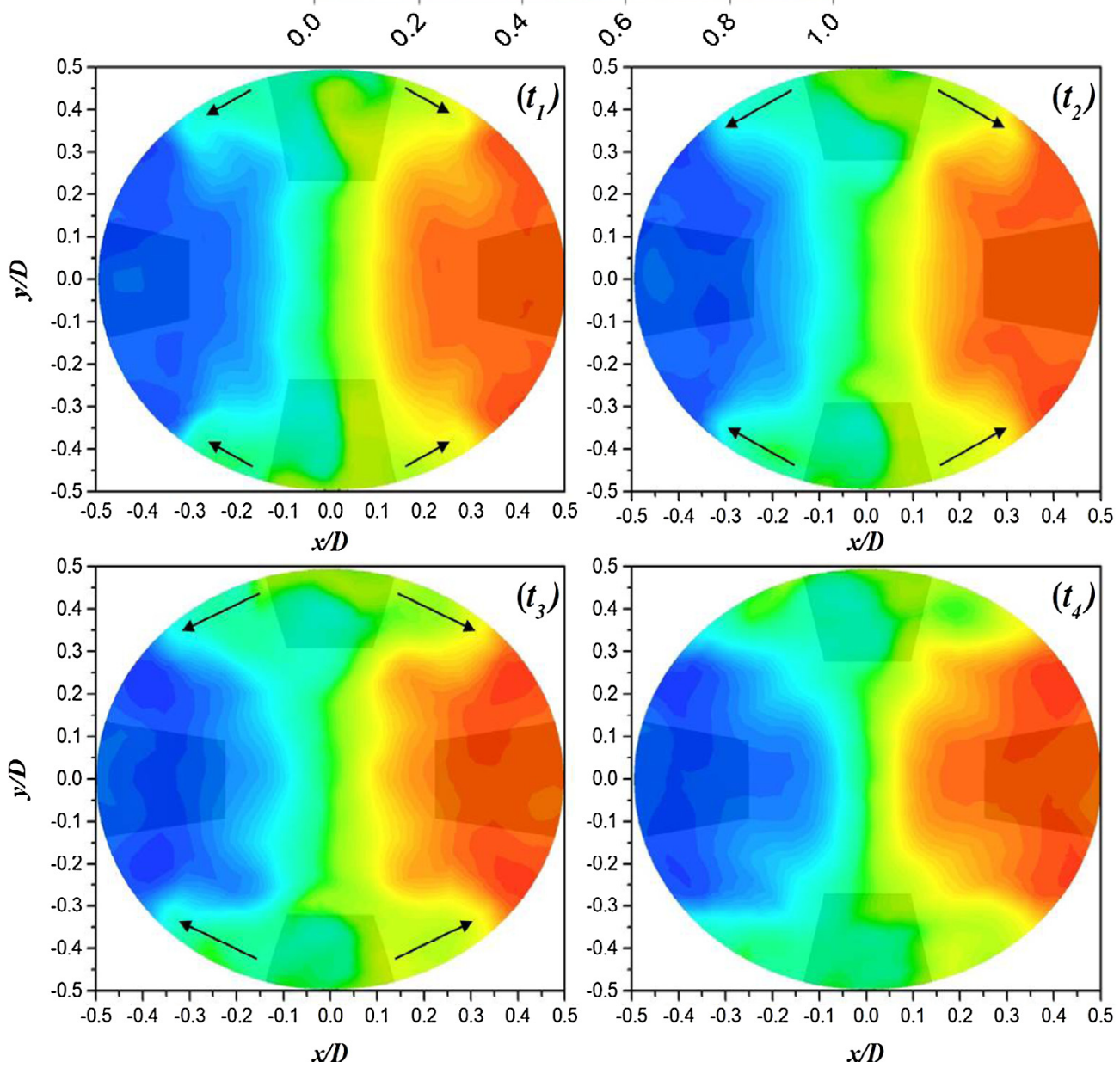

Fig. 19. Scalar field distribution at a cross sectional view $(z / d=3.33)$ for the FVG case at four time instants equally spaced by $0.04 \mathrm{~s}$. 


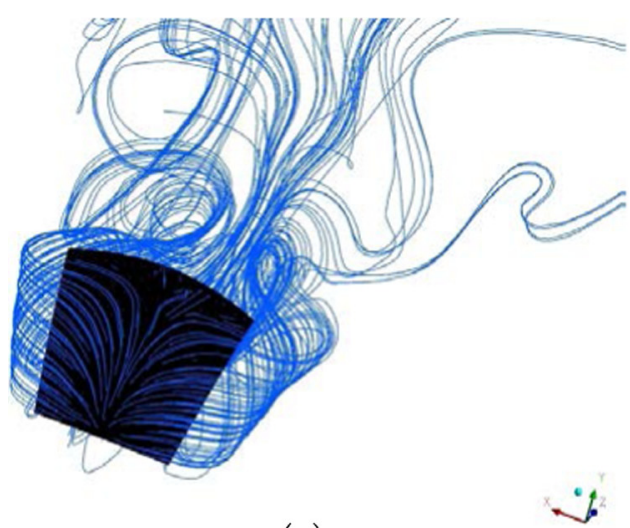

(a)

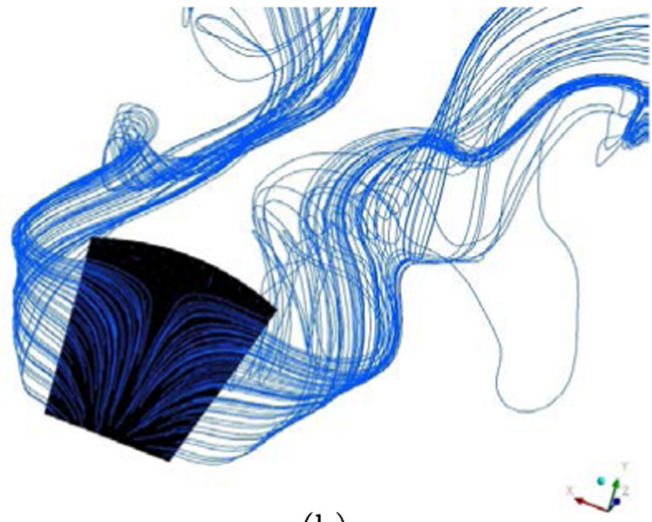

(b)

Fig. 20. Streamlines around tab En during two time instants: (a) $t_{1}$ and (b) (see Fig. 19) $t_{3}$.

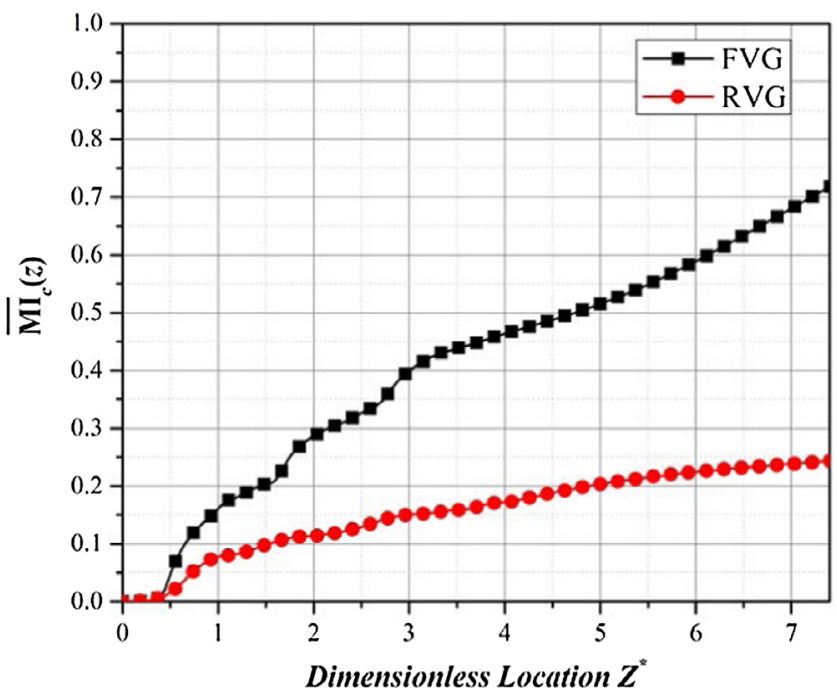

Fig. 21. Local mixing index $\left(\mathrm{MI}_{z}\right)$ along all the pipe length for the FVG and RVG cases.

while it is only 0.243 for the RVG case, corresponding to a $195 \%$ increase in the mixing performance.

\section{Conclusion}

In the present work, numerical simulation of fluid structure interaction is performed in order to assess the performance of flexible trapezoidal vortex generators (FVG) compared to rigid vortex generators (RVG) in terms of heat and mixing performances. The simulation for the FVG case is done by coupling ANSYS Fluent CFD solver, ANSYS Mechanical CSD solver and a third solver for mesh motion and remeshing operations in order to deal with the large displacement of the FVG.

Five rows of trapezoidal VG are placed in an HEV configuration and tangentially rotated by an angle of $45^{\circ}$ with respect to each other. After a transient period, the FVG undergo self sustained large displacement oscillation with a frequency approximately equal to their natural frequency. Meanwhile FVG located in rows B and D show small oscillation amplitudes, much more FVG interaction is obtained in rows A, C and E. This observation is particularly relevant with previous results obtained in two-dimensional FVG configurations (Ali et al., 2016) where three successive flaps were needed to reach a self-sustained oscillating system. For future full three-dimensional simulations, it is thus to be expected that adding a sixth tangentially rotated row F may have drastic effect on the oscillation of FVG located in rows B and D. Moreover, it is observed that for a given row, tabs located at the north and south positions always oscillate out of phase with tabs located at the east and west positions.

The flow patterns for the RVG and FVG cases have then been analyzed. The RVG case shows steady streamwise counterrotating vortex pairs (CVP) generated downstream the RVG due to the pressure difference between the high momentum core fluid and the low momentum fluid present in the wake region. These CVP play a crucial role as internal agitators between the hot fluid near the wall and the cold fluid in the core. Additional transverse vortices are identified in the wake of the tabs and can be considered to have a negative impact on heat transfer since the stagnant fluid stays trapped in this region and overheated regions remain there. Moreover, for the FVG case, the proper orthogonal decomposition (POD) technique has been applied to analyze the flow structures. The first four most energetic modes are used for the analysis as they contain most of the flow energy. FFT are performed on the temporal evolution of the POD coefficients and reveal a frequency peak equal to the frequency of tabs oscillation and corresponding to the fundamental frequency of the tabs. In this lock-in regime, the vortex shedding supplies energy to the structure instead of damping it resulting in a large amplitude oscillation. Nevertheless, from snapshot reconstruction based on the four most energetic modes the tabs oscillations are found to not only be responsible for vortices creation but are also responsible for operations of creation, destruction and dislocation of vortices. At the highest amplitudes positions, two high velocity shear layers wrap the two sides of the tabs and displace to the negative region behind the tabs. As the tabs oscillate in the opposite streamwise direction, these velocity shear layers are compressed in size as they disappear at the minimum amplitude. Then at this position, a horseshoe vortex forms and extends its two arms to each side of the tabs and into the wake region and may reach the center of the pipe. The major difference between the RVG and FVG case lies in the fact that the vortices are always located near the wall and at the tabs positions in the RVG case, while they are much more displaced towards the center and are either presented as packs in the north and south position or either in the east and west positions when the FVG configuration is considered.

In order to analyze the local performance of heat transfer between the two cases, the local Nusselt number is then determined. The FVG case is found to perform better in removing the heated regions at the wake of tabs and to exhibit also higher performance at the tabs positions where they undergo lower amplitude oscillations. At the positions where the tabs undergo high amplitude oscillations, the RVG case seems to perform better heat transfer. Conclusions drawn from this study are: as the tabs oscillate in 
the opposite streamwise direction, much of the flow structure and vortex intensity are degraded, as opposed to the RVG case where the tabs are always fixed to a specific high angle to the flow. Since the tabs oscillate out of phase at a given row, the vortices are either present at the north and south positions or east and west positions, which have a negative impact on heat transfer. However as the tabs oscillate in large amplitudes in rows $\mathrm{A}, \mathrm{C}$ and $\mathrm{E}$, they create high velocity flow, due to the increase in the blockage area which in return force the fluid to impact the next negligibly oscillating rows at high velocity (rows $B$ and $D$ ), hence displaying at the latter row location (B and D) a better convective heat transfer than the RVG case. In terms of global heat transfer analysis, the overall heat transfer enhancement with respect to empty pipe is about $118 \%$ for the FVG case and about $97 \%$ for the RVG case. Moreover, the thermal performance factor $\eta$ is evaluated and compared in both RVG and FVG cases to a straight pipe flow configuration. It is notably found to be below one for both cases: 0.995 for the RVG case and 0.987 for the FVG case, clearly displaying the increase in pressure drop penalty in both cases. This observation can be somewhat different from other studies where authors found that RVG can effectively improve heat transfer in HEV geometries for turbulent flow regimes. In the laminar flow investigated here, the authors believe that good thermal performance can be achieved using optimized designs of FVG. No optimization study have been performed in the time being but could be achieved using experimental approach for example in order to overcome the computationally expensive simulations due to fluid-structure interaction problem.

Finally, the mixing performance is compared between the RVG and FVG cases by analyzing the mixture quality of a passive scalar equally divided into the right and left side of the pipe at its initial stage ( $c=1$ for $x>0$ and $c=0$ for $x<0)$. It is observed that the FVG greatly help in enhancing the mixing performance by stretching and engulfing the scalars into each initially separated halves since the vortices of the FVG case are much more dislocated to the center of the pipe and at the position of the separation zone between the two scalars. This is shown by the wider high quality mixture zone appearing at the center. The oscillations of the tabs, especially at the opposite streamwise direction, stretch out the two scalars to both lateral sides of the tabs and increase the mixture quality. The local mixing index $\operatorname{MI}_{c}(z)$ is then evaluated and the FVG case displays higher values all along the pipe length than the RVG case and can thus lead to more compact and shorter mixers/reactors. The mixture quality enhancement is eventually increased by about $195 \%$ in the FVG configuration compared to the RVG case.

\section{Appendix A. Supplementary material}

Supplementary data associated with this article can be found, in the online version, at http://dx.doi.org/10.1016/j.ces.2016.12.039.

\section{References}

Ahmed, H.E., Mohammed, H.A., Yusoff, M.Z., 2012. An overview on heat transfer augmentation using vortex generators and nanofluids: approaches and applications. Renew. Sustain. Energy Rev. 16, 5951-5993.
Akcabay, D.T., Young, Y.L., 2012. Hydroelastic response and energy harvesting potential of flexible piezoelectric beams in viscous flow. Phys. Fluids 24 054106.

Ali, S., Habchi, C., Menanteau, S., Lemenand, T., Harion, J.-L., 2015. Heat transfer and mixing enhancement by free elastic flaps oscillation. Int. J. Heat Mass Transf. 85, 250-264.

Ali, S., Menanteau, S., Habchi, C., Lemenand, T., Harion, J.-L., 2016. Heat transfer and mixing enhancement by using multiple freely oscillating flexible vortex generators. Appl. Therm. Eng. 105, 276-289.

Allison, C., Dally, B., 2007. Effect of a delta-winglet vortex pair on the performance of a tube-fin heat exchanger. Int. J. Heat Mass Transf. 50, 5065-5072.

ANSYS Academic Research, 2015. Release 15.0, Help System, User's Guide, ANSYS, Inc.

Anxionnaz, Z., Cabassud, M., Gourdon, C., Tochon, P., 2008. Heat exchanger/reactors (hex reactors): concepts, technologies: state-of-the-art. Chem. Eng. Process. 47, 2029-2050.

Bos, F.M., 2010. Numerical Simulations of Flapping Foil and Wing Aerodynamics. Phd. Technical University of Delft.

Celik, I.B., Ghia, U., Roache, P.J., Freitas, C.J., Coleman, H., Raad, P.E., 2008. Procedure for estimation and reporting of uncertainty due to discretization in CFD applications. J. Fluids Eng. 130, 0780011-0780014.

Donea, J., Huerta, A., Ponthot, J.P., Rodriguez-Ferran, A., 2004. Arbitrary Lagrangian Eulerian Methods. John Wiley \& Sons, Ltd.

Dong, S., Meng, H., 2004. Flow past a trapezoidal tab. J. Fluid Mech. 510, 219-242.

Galpin, P.F., Broberg, R.B., Hutchinson, B.R., 1995. Three-dimensional Navier Stokes predictions of steady-state rotor/stator interaction with pitch change. In: 3rd Annual Conference of the CFD, Society of Canada, Banff, Alberta, Canada, June 25-27. Advanced Scientific Computing Ltd.

Habchi, C., Lemenand, T., Della Valle, D., Peerhossaini, H., 2010a. Turbulent mixing and residence time distribution in novel multifunctional heat exchangersreactors. Chem. Eng. Process. 49, 1066-1075.

Habchi, C., Lemenand, T., Della Valle, D., Peerhossaini, H., 2010b. Turbulence behavior of artificially generated vorticity. J. Turbul. 11, 1-18.

Habchi, C., Russeil, S., Bougeard, D., Harion, J.-L., Lemenand, T., Della Valle, D. Peerhossaini, H., 2012. Enhancing heat transfer in vortex generator-type multifunctional heat exchangers. Appl. Therm. Eng. 38, 14-25.

Habchi, C., Harion, J.-L., Russeil, S., Bougeard, D., Hachem, F., Elmarakbi, A., 2013. Chaotic mixing by longitudinal vorticity. Chem. Eng. Sci. 104, 439-450.

Huang, L., 1995. Flutter of cantilevered plates in axial flow. J. Fluids Struct. 9, 127 147.

Lambert, R.A., Rangel, R.H., 2010. The role of elastic flap deformation on fluid mixing in a microchannel. Phys. Fluids 22, 1-12.

Lei, Y.-G., He, Y.-L., Tian, L.-T., Chu, P., Tao, W.-Q., 2010. Hydrodynamics and heat transfer characteristics of a novel heat exchanger with delta-winglet vortex generators. Chem. Eng. Sci. 65, 1551-1562.

Le Tallec, P., Mouro, J., 2001. Fluid structure interaction with large structural displacements. Comput. Methods Appl. Mech. Eng. 190, 3039-3067.

MATLAB, version 7.10.0, R2010a. The MathWorks Inc., Natick, Massachusetts.

Michelin, S., Doaré, O., 2013. Energy harvesting efficiency of piezoelectric flags in axial flows. J. Fluid Mech. 714, 489-504.

Promvonge, P., Thianpong, C., 2008. Thermal performance assessment of turbulent channel flows over different shaped ribs. Int. Commun. Heat Mass Transf. 35, 1327-1334.

Shi, J., Hu, J., Schafer, S., Chen, C., 2014. Numerical study of heat transfer enhancement of channel via vortex-induced vibration. Appl. Therm. Eng. 70, 838-845.

Sirovich, L., Kirby, M., 1987. Low-dimensional procedure for the characterization of human faces. JOSA A 4, 519-524.

Soti, A.K., Bhardwaj, R., Sheridan, J., 2015. Flow-induced deformation of a flexible thin structure as manifestation of heat transfer enhancement. Int. J. Heat Mass Transf. 84, 1070-1081.

Tukovic, Z., Jasak, H., 2007. Updated lagrangian finite volume solver for large deformation dynamic response of elastic body. Trans. FAMENA 31, 1-16.

Turek, S., Hron, J., 2006. Proposal for Numerical Benchmarking of Fluid-structure Interaction Between an Elastic Object and Laminar Incompressible Flow. Springer.

Williamson, C.H.K., Govardhan, R., 2004. Vortex-induced vibrations. Annu. Rev Fluid Mech. 36, 413-455.

Yang, Y.-T., Chen, C.-H., 2008. Numerical simulation of turbulent fluid flow and heat transfer characteristics of heated blocks in the channel with an oscillating cylinder. Int. J. Heat Mass Transf. 51, 1603-1612. 